Bull. Soc. math. France

134 (2), 2006, p. 287-325

\title{
INVARIANTS OF REAL SYMPLECTIC FOUR-MANIFOLDS OUT OF REDUCIBLE AND CUSPIDAL CURVES
}

\author{
By Jean-Yves Welschinger
}

\begin{abstract}
We construct invariants under deformation of real symplectic fourmanifolds. These invariants are obtained by counting three different kinds of real rational $J$-holomorphic curves which realize a given homology class and pass through a given real configuration of (the appropriate number of) points. These curves are cuspidal curves, reducible curves and curves with a prescribed tangent line at some real point of the configuration. They are counted with respect to some sign defined by the parity of their number of isolated real double points and in the case of reducible curves, with respect to some mutiplicity. In the case of the complex projective plane equipped with its standard symplectic form and real structure, these invariants coincide with the ones previously constructed in [11]. This leads to a relation between the count of real rational $J$-holomorphic curves done in [11] and the count of real rational reducible $J$-holomorphic curves presented here.
\end{abstract}

Texte reçu le 5 avril 2005, accepté le 26 octobre 2005.

Jean-Yves Welschinger, École Normale Supérieure de Lyon, Unité de Mathématiques Pures et Appliquées, UMR CNRS 5669, 46, allée d'Italie 69364, Lyon Cedex 07 (France). E-mail : jwelschi@umpa.ens-lyon.fr

2000 Mathematics Subject Classification. - 53D45, 14N35, 14N10, 14 P99.

Key words and phrases. - Real symplectic manifold, rational curve, enumerative geometry. 
RÉSUMÉ (Courbes réductibles, cuspidales et invariants des variétés symplectiques réelles de dimension quatre)

Nous construisons des invariants par déformation des variétés symplectiques réelles de dimension quatre. Ces invariants sont obtenus en comptant trois différents types de courbes $J$-holomorphes rationnelles réelles qui réalisent une classe d'homologie donnée et passent par une configuration réelle donnée d'un nombre (adéquat) de points. Ces courbes sont des courbes cuspidales, réductibles et des courbes ayant une tangente prescrite en l'un des points de la configuration. Elles sont comptées en fonction d'un signe qui dépend de la parité du nombre de leurs points doubles réels isolés et, dans le cas des courbes réductibles, en fonction d'une multiplicité. Dans le cas du plan projectif complexe muni de ses formes symplectiques et structures réelles standards, ces invariants coincident avec ceux précédemment construits dans [11]. Ceci mène à une relation entre le comptage de courbes $J$-holomorphes rationnelles réelles réalisé dans [11] et le comptage de courbes $J$-holomorphes rationnelles réductibles réelles présenté ici.

\section{Contents}

Introduction and statement of the results 288

1. Moduli space of real rational cuspidal pseudo-

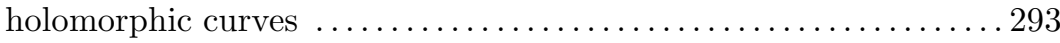

2. Study of degenerated order two cuspidal points $\ldots \ldots \ldots \ldots \ldots 305$

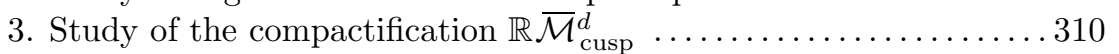

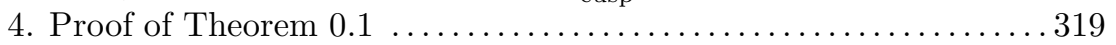

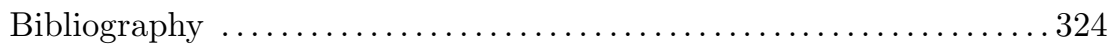

\section{Introduction and statement of the results}

Let $\left(X, \omega, c_{X}\right)$ be a real symplectic four-manifold, that is a triple made of a smooth compact four-manifold $X$, a symplectic form $\omega$ on $X$ and an involution $c_{X}$ on $X$ such that

$$
c_{X}^{*} \omega=-\omega .
$$

The fixed point set of $c_{X}$ is called the real part of $X$ and is denoted by $\mathbb{R} X$.

A large source of examples is provided by smooth projective surfaces defined by a system of polynomials with real coefficients, the symplectic form is then the restriction of the Fubini-Study form of the ambiant projective space, and the real structure is the restriction of its complex conjugation. Note that the real locus $\mathbb{R} X$ is assumed to be non empty here so that it is a smooth lagrangian surface of $(X, \omega)$.

With every such real symplectic four-manifold comes some function

$$
\chi: d \in H_{2}(X ; \mathbb{Z}) \longmapsto \chi^{d}(T) \in \mathbb{Z}\left[T_{1}, \ldots, T_{N}\right],
$$

TOME $134-2006-\mathrm{N}^{\mathrm{O}} 2$ 
where $N$ denotes the number of connected components of the real locus of the manifold. This function has been constructed in [11] by exctracting integer valued invariants - the coefficients of the polynomial $\chi^{d}(T)$ - from the following problem of real enumerative geometry: how many real rational curves do realize the homology class $d$ and pass through the adequate number of points?

Remember that for this problem to make sense, we introduce an auxiliary generic almost complex structure $J$, that is a complex structure on the tangent bundle $T X$, and we count real rational $J$-holomorphic curves, that is immersed two-dimensional spheres which are preserved by the involution $c_{X}$ and whose tangent planes are invariant under $J$. The adequate number of points is then the expected dimension of this space of real rational $J$-holomorphic curves, that is $c_{1}(X) d-1$, where $c_{1}(X)$ is the first Chern class of the manifold $(X, \omega)$.

Remember that all of these finitely many curves are images of $\mathbb{Z} / 2 \mathbb{Z}$ equivariant immersions $u:\left(\mathbb{C} P^{1}\right.$, conj $) \rightarrow\left(X, c_{X}\right)$ and the above mentionned invariants are obtained by counting these curves with respect to some sign \pm 1 determined by the parity of the number of pairs of complex conjugated points in the set $u^{-1}(\mathbb{R} X)$.

For example, the cubic planar real rational curve parameterized by $t \in \mathbb{C} \mapsto$ $\left(t^{2}, t^{3}+\epsilon t\right)$ is counted positively if $\epsilon<0$ and negatively if $\epsilon>0$ since $u^{-1}(\mathbb{R} X)$ then contains $\{ \pm i \sqrt{\epsilon}\}$, and the pure imaginary planar conic with affine equation $x^{2}+y^{2}=-1$ is a real rational curve, but not the image of a $\mathbb{Z} / 2 \mathbb{Z}$-equivariant immersion $u:\left(\mathbb{C} P^{1}\right.$, conj $) \rightarrow\left(X, c_{X}\right)$ since its real part is empty.

Remember finally that if we do not obtain a unique invariant as the GromovWitten invariant in the complex case, it is due to the fact the integers we obtain depend on the number of pairs of complex conjugated points in the chosen configuration of $c_{1}(X) d-1$ points as well as on the distribution of the remaining points in the different connected components of the real part.

The existence of these invariants raises various questions. Are there analog invariants in higher dimensions? Of which problems of real enumerative geometry is it possible to extract some integer valued invariants? Note that such invariants then bound from below the number of real solutions of the given problem, see Corollary 2.2 of [11]. Does some recursive formula similar to the one obtained by M. Kontsevich for the Gromov-Witten invariants exist?

The works [12] and [10] provide some positive answer to the first question. The present paper, as well as [9] which can be considered as a continuation of this work, is devoted to the study of the next two questions. The problem addressed in [9] is to replace one point condition in the above problem by one tangency condition with some given curve $L$ in the real part $\mathbb{R} X$, as in the classical problem of counting real planar conics tangent to five generic real conics for example. It is proven in [9] that some integer valued invariants can indeed be extracted from this problem, but this requires to take into account other kinds of curves which appear in generic 1-parameter families of curves, 
namely two components reducible curves, cuspidal curves and curves with some prescribed tangent line at one point of the configuration (or equivalently from Proposition 3.4 of [11], curves having one double point at some point of the configuration). The present paper is actually devoted to the case where $L$ is empty. In this case, only the three terms we have just mentionned occur and indeed they hide some integer valued invariants, see Theorem 0.1. Moreover, these new invariants can be compared with the ones of [11], see Proposition 0.3 below, leading to some relation between the count of generic real rational curves of [11] with the one of real reducible curves done here. However, this relation does not lead to some recursive formula similar to the one obtained in the complex case by M. Kontsevich, see Remark 0.4 below. Note that since the preprint version of this paper and of [9] have appeared, progress has been made on the questions of computation or finding recursion formulas, see [13] and Remark 3 therein.

Let us now come to the precise formulation of the main results of this paper.

We label the connected components of the real part by $(\mathbb{R} X)_{1}, \ldots,(\mathbb{R} X)_{N}$.

Let $\ell \gg 1$ be an integer large enough and $\mathcal{J}_{\omega}$ be the space of almost complex structures of $X$ which are tamed by $\omega$ and of class $C^{\ell}$. Let $\mathbb{R} \mathcal{J}_{\omega}$ be the subspace of $\mathcal{J}_{\omega}$ made of almost complex structures for which the involution $c_{X}$ is $J$-antiholomorphic. These two spaces are separable Banach manifolds which are non empty and contractible (see $\S 1.1$ of $[11]$ for the real case).

Let $d \in H_{2}(X ; \mathbb{Z})$ be a homology class satisfying $c_{1}(X) d>1$ and set

$$
\nu=c_{1}(X)-2 \text {. }
$$

Let $\underline{x}=\left(x_{1}, \ldots, x_{\nu}\right) \in X^{\nu}$ be a real configuration of $\nu$ distinct points of $X$, that is an ordered subset of distinct points of $X$ which is globally invariant under $c_{X}$. For $j \in\{1, \ldots, N\}$, we denote by $r_{j}$ the number of points in the configuration $\underline{x}$ that are located in the component $(\mathbb{R} X)_{j}$ and we set

$$
r=\left(r_{1}, \ldots, r_{N}\right)
$$

so that the $N$-tuple $r$ encodes the equivariant isotopy class of $\underline{x}$. We will assume throughout the paper that $r \neq(0, \ldots, 0)$, see Remark 3.5.

Finally, denote by $I$ the subset of those $i \in\{1, \ldots, \nu\}$ for which $x_{i}$ is fixed by the involution $c_{X}$.

For each $i \in I$, choose a line $T_{i}$ in the tangent plane $T_{x_{i}} \mathbb{R} X$.

Then, for a generic choice of $J \in \mathbb{R} \mathcal{J}_{\omega}$, there are only finitely many real rational $J$-holomorphic curves which realize the homology class $d$, pass through $\underline{x}$ and are cuspidal. Moreover, these curves are all irreducible and have only transversal double points as well as a unique real ordinary cusp as singularities.

Denote by $\operatorname{Cusp}^{d}(J, \underline{x})$ this finite set of cuspidal curves.

Likewise, there are only finitely many real rational $J$-holomorphic curves which realize the homology class $d$, pass through $\underline{x}$ and are reducible. Moreover,

TOME $134-2006-\mathrm{N}^{\mathrm{O}} 2$ 
these curves have only two irreducible components and only transversal double points as singularities.

Denote by $\mathcal{R} e d^{d}(J, \underline{x})$ this finite set of reducible curves.

Note that since $I \neq \varnothing$, both irreducible components of such curves are real. Indeed, they would otherwise be exchanged by the involution $c_{X}$ and would intersect the real locus at only finitely many points. The condition to pass through a point of $I$ would then cost two degrees of liberty instead of one so that generically such curves do not appear. Finally, there are only finitely many real rational $J$-holomorphic curves which realize the homology class $d$, pass through $\underline{x}$ and whose tangent line at some point $x_{i}, i \in I$, is $T_{i}$. Moreover, the point $x_{i}$ having this property is then unique and these curves are all irreducible with only transversal double points as singularities.

Denote by $\mathcal{T a n}^{d}(J, \underline{x})$ this finite set of rational curves.

Note that if $C \in \mathcal{C} u s p^{d}(J, \underline{x}) \cup \operatorname{Red}^{d}(J, \underline{x}) \cup \mathcal{T}^{d}{ }^{d}(J, \underline{x})$, then all the singularities of $C$ are disjoint from $\underline{x}$.

Following [11], we define the mass of $C$ and denote by $m(C)$ its number of real isolated double points.

Here, a real double point is said to be isolated when it is the local intersection of two complex conjugated branches, whereas it is said to be non isolated when it is the local intersection of two real branches.

If $C$ belongs to $\operatorname{Red}^{d}(J, \underline{x})$ and $C_{1}, C_{2}$ denote its irreducible components, then we define the multiplicity of $C$, and denote by mult $(C)$, the number of real intersection points between $C_{1}$ and $C_{2}$, that is the cardinality of $\mathbb{R} C_{1} \cap \mathbb{R} C_{2}$.

We then set

$$
\Gamma_{r}^{d}(J, \underline{x})=\sum_{C \in \mathcal{C} u s p^{d}(J, \underline{x}) \cup \mathcal{T} a n^{d}(J, \underline{x})}(-1)^{m(C)}-\sum_{C \in \mathcal{R e d}^{d}(J, \underline{x})}(-1)^{m(C)} \operatorname{mult}(C) .
$$

THEOREM 0.1. - Let $\left(X, \omega, c_{X}\right)$ be a real symplectic four-manifold and

$$
d \in H_{2}(X ; \mathbb{Z}) \quad \text { be such that } c_{1}(X) d>1, c_{1}(X) d \neq 4 \text {. }
$$

The connected components of $\mathbb{R} X$ are labeled by $(\mathbb{R} X)_{1}, \ldots,(\mathbb{R} X)_{N}$. Let $\underline{x} \subset X$ be a real configuration of $c_{1}(X) d-2$ distinct points, $r_{j}$ be the cardinality of $\underline{x} \cap(\mathbb{R} X)_{j}$ and $r=\left(r_{1}, \ldots, r_{N}\right)$. Finally, let $J \in \mathbb{R} \mathcal{J}_{\omega}$ be generic enough so that the integer $\Gamma_{r}^{d}(J, \underline{x})$ is well defined. Then, this integer $\Gamma_{r}^{d}(J, \underline{x})$ neither depends on the choice of J, nor on the choice of $\underline{x}$.

(The condition $c_{1}(X) d \neq 4$ is to avoid appearance of multiple curves, see Remark 1.10.)

From this theorem, the integer $\Gamma_{r}^{d}(J, \underline{x})$ can be denoted without ambiguity by $\Gamma_{r}^{d}$, and when it is not well defined, we set $\Gamma_{r}^{d}=0$. We then denote by $\Gamma^{d}(T)$ the generating function

$$
\sum_{r \in \mathbb{N}^{N}} \Gamma_{r}^{d} T^{r} \in \mathbb{Z}\left[T_{1}, \ldots, T_{N}\right]
$$

BULletin DE LA SOCIÉtÉ MATHÉMATIQUE DE FRANCE 
where $T^{r}=T_{1}^{r_{1}} \cdots T_{N}^{r_{N}}$. This polynomial function is of the same parity as $c_{1}(X) d$ and each of its monomial actually only depends on one indeterminate. Indeed, the real part of any real rational curve is connected and thus it cannot interpolate points in different connected components of $\mathbb{R} X$. This in fact implies that any symplectic four-manifold having a real structure with disconnected real locus must have even genus zero Gromov-Witten invariants as soon as the number of point conditions is greater than one. It follows from Theorem 0.1 that the function

$$
\Gamma: d \in H_{2}(X ; \mathbb{Z}) \longmapsto \Gamma^{d}(T) \in \mathbb{Z}[T]
$$

only depends of the real symplectic four-manifold $\left(X, \omega, c_{X}\right)$. Moreover, it is invariant under deformation of this real symplectic four-manifold, that is if $\omega_{t}$ is a continuous family of symplectic forms on $X$ for which $c_{X}^{*} \omega_{t}=-\omega_{t}$, then this function is the same for all $\left(X, \omega_{t}, c_{X}\right)$. As an application of this invariant, we obtain the following lower bounds in real enumerative geometry.

COROLlaRY 0.2. - Under the hypothesis of Theorem 0.1 , the integer $\left|\Gamma_{r}^{d}\right|$ provides a lower bound for the cardinality of the weighted set

$$
\mathcal{C u s p}^{d}(J, \underline{x}) \cup \mathcal{R e d}^{d}(J, \underline{x}) \cup \mathcal{T}^{d}(J, \underline{x}),
$$

independently of the choice of a generic $J \in \mathbb{R} \mathcal{J}_{\omega}$ and $\underline{x}$.

The non triviality of the invariant $\Gamma_{r}^{d}$ is guaranteed by the following proposition, see Corollary 1.4 of [9].

Proposition 0.3. - Let $\left(X, \omega, c_{X}\right)$ be the complex projective plane equipped with its standard symplectic form and real structure, so that $H_{2}(X ; \mathbb{Z})$ is canonically isomorphic to $\mathbb{Z}$. Let $r, d$ be integers satisfying $d \geq 2$ and $1 \leq r \leq 3 d-2$. Then $\Gamma_{r}^{d}=\chi_{r+1}^{d}$.

REMARK 0.4. - Remember that the integer $\chi_{r+1}^{d}$ has been defined in [11] by counting the number of real rational $J$-holomorphic curves of degree $d$ which pass through $3 d-1$ points with respect to the parity of their mass. Likewise, the integer $\Gamma_{r}^{d}$ has just been defined above by counting three kinds of curves which appear in codimension 1 in the space of real rational $J$-holomorphic curves of degree $d$. In particular, one of the three kinds is reducible curves. The equality given by Proposition 0.3 thus provides a relation between the invariant $\chi_{r+1}^{d}$ and an analogous sum over all real reducible curves passing through $3 d-2$ points. That is precisely what one would need to provide a recursion formula similar to the one obtained by Kontsevich to compute the rational Gromov-Witten invariants of $\mathbb{C} P^{2}$, see [4]. However, the reducible curves are counted here with respect to some real multiplicity which is not under control, and likewise, there are two other kinds of curves which are counted in the expression of $\Gamma_{r}^{d}$ which we do not control.

TOME $134-2006-\mathrm{N}^{\mathrm{O}} 2$ 
The paper is organized as follows. The first paragraph is devoted to the construction of the moduli space $\mathbb{R} \mathcal{M}_{\text {cusp }}^{d}$ of real rational cuspidal pseudoholomorphic curves which realize the homology class $d$. This space is equipped with a projection $\pi_{\mathbb{R}}: \mathbb{R} \mathcal{M}_{\text {cusp }}^{d} \rightarrow \mathbb{R} \mathcal{J}_{\omega} \times \mathbb{R}_{\tau} X^{\nu}$. The critical points of $\pi_{\mathbb{R}}$ as well as its lack of properness are discussed there. The second paragraph is entirely devoted to the study of one particular type of critical points of $\pi_{\mathbb{R}}$, namely those arising from curves having a degenerated cuspidal point. The third paragraph is devoted to the study of the Gromov compactification $\overline{\mathbb{R}}_{\text {cusp }}^{d}$ of $\mathbb{R} \mathcal{M}_{\text {cusp }}^{d}$. Finally, the fourth paragraph is devoted to the proof of Theorem 0.1.

Acknowledgements. - This work was initiated during my stay at the Mathematical Sciences Research Institute in spring 2004. I would like to acknowledge MSRI for the excellent working conditions it provided to me.

\section{Moduli space of real rational cuspidal pseudo- holomorphic curves}

Let $d \in H_{2}(X ; \mathbb{Z})$ be such that

$$
\left(c_{X}\right)_{*} d=-d \quad \text { and } \quad c_{1}(X) d>1, c_{1}(X) d \neq 4 .
$$

Let $\tau$ be an order 2 permutation of the set $\{1, \ldots, \nu\}$, where $\nu=c_{1}(X) d-2$, and $c_{\tau}:\left(x_{1}, \ldots, x_{\nu}\right) \in X^{\nu} \mapsto\left(c_{X}\left(x_{\tau(1)}\right), \ldots, c_{X}\left(x_{\tau(\nu)}\right)\right) \in X^{\nu}$ be the associated real structure of $X^{\nu}$. The fixed point set of $c_{\tau}$ is denoted by $\mathbb{R}_{\tau} X^{\nu}$.

\subsection{Moduli space $\mathcal{P}_{\text {cusp }}^{*}$ of cuspidal pseudo-holomorphic maps}

Let $S$ be an oriented sphere of dimension 2 and $\mathcal{J}_{S}$ be the space of complex structures of class $C^{\ell}$ of $S$ which are compatible with its orientation. Let $\underline{z}=\left(z_{1}, \ldots, z_{\nu}\right) \in S^{\nu}$ be an ordered set of $\nu$ distinct points of $S$. Let $\nabla$ be a torsion free connection on $T X$ which is invariant under $c_{X}$. We set

$$
\begin{aligned}
\mathcal{P}=\left\{\left(u, J_{S}, J, \underline{x}\right) \in L^{k, p}(S, X) \times \mathcal{J}_{S} \times \mathcal{J}_{\omega} \times X^{\nu} \mid\right. \\
\left.u_{*}[S]=d, u(\underline{z})=\underline{x}, \mathrm{~d} u+J \circ \mathrm{d} u \circ J_{S}=0\right\},
\end{aligned}
$$

where $1 \ll k \ll \ell$ is large enough and $p>2$. Let $\mathcal{P}^{*} \subset \mathcal{P}$ be the space of non multiple pseudo-holomorphic maps, that is the space of quadruples $\left(u, J_{S}, J, \underline{x}\right)$ for which $u$ cannot be written $u^{\prime} \circ \Phi$ where $\Phi: S \rightarrow S^{\prime}$ is a non trivial ramified covering and $u^{\prime}: S^{\prime} \rightarrow X$ a pseudo-holomorphic map.

Remember that $\mathcal{P}^{*}$ is a separable Banach manifold of class $C^{\ell-k}$ (see [5], Proposition 3.2.1) with tangent bundle

$$
\begin{array}{r}
T_{\left(u, J_{S}, J, \underline{x}\right)} \mathcal{P}^{*}=\left\{\left(v, \dot{J}_{S}, \dot{j}, \underline{\dot{x}}\right) \in T_{\left(u, J_{S}, J, \underline{x}\right)}\left(L^{k, p}(S, X) \times \mathcal{J}_{S} \times \mathcal{J}_{\omega} \times X^{\nu}\right)\right. \\
\left.\mid v(\underline{z})=\underline{\dot{x}}, D v+J \circ \mathrm{d} u \circ \dot{J}_{S}+\dot{J} \circ \mathrm{d} u \circ J_{S}=0\right\} .
\end{array}
$$

BULletin DE LA SOCiÉtÉ MATHÉMATIQUE DE FRANCE 
Here, $T_{u} L^{k, p}(S, X)=\left\{v \in L^{k, p}\left(S, E_{u}\right)\right\}$ where $E_{u}=u^{*} T X$ and

$$
\begin{aligned}
D: v \in L^{k, p}\left(S, E_{u}\right) & \\
& \longmapsto \nabla v+J \circ \nabla v \circ J_{S}+\nabla_{v} J \circ \mathrm{d} u \circ J_{S} \in L^{k-1, p}\left(S, \Lambda^{0,1} S \otimes E_{u}\right)
\end{aligned}
$$

is the associated Gromov operator (see [5], Proposition 3.1.1). Let

$$
\begin{aligned}
\mathcal{P}_{\text {cusp }}^{*} & =\left\{\left(\left(u, J_{S}, J, \underline{x}\right), z_{c}\right) \in \mathcal{P}^{*} \times S \mid \mathrm{d}_{z_{c}} u=0\right\}, \\
\mathcal{P}_{\text {hocusp }}^{*} & =\left\{\left(\left(u, J_{S}, J, \underline{x}\right), z_{c}\right) \in \mathcal{P}_{\text {cusp }}^{*}|\nabla \mathrm{d} u|_{z_{c}}=0\right\}
\end{aligned}
$$

be the subspace of maps having a higher order cuspidal point at $z_{c}$.

Proposition 1.1. - The space $\mathcal{P}_{\text {cusp }}^{*}$ is a separable Banach manifold of class $C^{\ell-k}$ with tangent bundle

$$
\begin{array}{r}
T_{\left(\left(u, J_{S}, J, \underline{x}\right), z_{c}\right)} \mathcal{P}_{\text {cusp }}^{*}=\left\{\left(v, \dot{J}_{S}, \dot{J}, \underline{\dot{x}}, \dot{z}_{c}\right) \in T_{\left(u, J_{S}, J, \underline{x}\right)} \mathcal{P}^{*} \times T_{z_{c}} S\right. \\
\left.\mid \nabla v_{z_{c}}+\nabla_{\dot{z}_{c}} \mathrm{~d} u=0\right\}
\end{array}
$$

The space $\mathcal{P}_{\text {hocusp }}^{*}$ is a separable Banach submanifold of $\mathcal{P}_{\text {cusp }}^{*}$ of class $C^{\ell-k}$ and real codimension four.

Proof. - The proof is analogous to the one of Proposition 2.7 of [11]; we just recall a sketch of it. Denote by $F$ the vector bundle over $\mathcal{P}^{*} \times S$ whose fibre over $\left(\left(u, J_{S}, J, \underline{x}\right), z_{c}\right)$ is the vector space $T_{z_{c}}^{*} S \otimes T_{u\left(z_{c}\right)} X$. In particular, the restriction of $F$ over $\left\{\left(u, J_{S}, J, \underline{x}\right)\right\} \times S$ is the bundle $T^{*} S \otimes_{\mathbb{C}} u^{*} T X$. From Proposition 3.2.1 of [5], the bundle $F$ is of class $C^{\ell-k}$ since trivialization maps depend $C^{\ell-k}$-smoothly on $u$ and $C^{\ell-2}$-smoothly on $z_{c}, u$ being of class $C^{\ell}$ from [5], Theorem B.4.1. The section $\mathrm{d}_{z_{c}} u$ of $F$ is of class $C^{\ell-k}$ and vanishes transversely from Lemma 2.6 of [11]. The first part of the proposition follows and the second part can be proved along the same lines.

Remember that if $o\left(z_{c}\right)$ denotes the vanishing order of $\mathrm{d} u$ at $z_{c}$, then the jet of $u$ at the order $2 o\left(z_{c}\right)+1$ is a well-defined complex polynomial (see [8], Proposition 3). The subspace $\mathcal{P}_{\text {hocusp }}^{*}$ is precisely made of maps $u$ for which $o\left(z_{c}\right)>1$. When $o\left(z_{c}\right)=1$, this complex polynomial can be written

$$
j_{2}(u)\left(z-z_{c}\right)^{2}+j_{3}(u)\left(z-z_{c}\right)^{3}
$$

where $j_{2}(u), j_{3}(u) \in T_{u\left(z_{c}\right)} X, z$ is a complex coordinate of $\left(S, J_{S}\right)$ in a neighbourhood of $z_{c}$ and $j_{2}(u) \neq 0$ generates the tangent line of $u$ at the cuspidal point $u\left(z_{c}\right)$. The cuspidal points for which $j_{3}(u)$ is colinear to $j_{2}(u)$ are said to be degenerated. They will be studied in detail in $\S 2$.

TOME $134-2006-\mathrm{N}^{\mathrm{O}} 2$ 
1.2. Normal sheaf. - Remember that the $\mathbb{C}$-linear part of the Gromov operator $D$ is some Cauchy-Riemann-operator denoted by $\bar{\partial}$. The latter induces a holomorphic structure on the bundle $E_{u}=u^{*} T X$ which turns the morphism $\mathrm{d} u: T S \rightarrow E_{u}$ into an injective homomorphism of analytic sheaves (see [3], Lemma 1.3.1). Likewise, the $\mathbb{C}$-antilinear part of $D$ is some order 0 operator denoted by $R$ and defined by the formula

$$
R_{\left(u, J_{S}, J, \underline{x}\right)}(v)=N_{J}(v, \mathrm{~d} u),
$$

where $N_{J}$ is the Nijenhuis tensor of $J$. Denote by $\mathcal{N}_{u}$ the quotient sheaf $\mathcal{O}_{S}\left(E_{u}\right) / \mathrm{d} u\left(\mathcal{O}_{S}(T S)\right)$ so that it fits in the following exact sequence of analytic sheaves

$$
0 \rightarrow \mathcal{O}_{S}(T S) \longrightarrow \mathcal{O}_{S}\left(E_{u}\right) \longrightarrow \mathcal{N}_{u} \rightarrow 0 .
$$

As soon as $\left(\left(u, J_{S}, J, \underline{x}\right), z_{c}\right)$ belongs to $\mathcal{P}_{\text {cusp }}^{*}$, this exact sequence extends to

$$
0 \rightarrow \mathcal{O}_{S}(T S) \otimes \mathcal{O}_{S}\left(z_{c}\right) \longrightarrow \mathcal{O}_{S}\left(E_{u}\right) \longrightarrow \mathcal{N}_{u}^{z_{c}} \rightarrow 0
$$

where $\mathcal{N}_{u}^{z_{c}}$ is a quotient of the sheaf $\mathcal{N}_{u}$. We denote in this case by $\mathbb{C}_{z_{c}}$ the skyscraper subsheaf

$$
\mathrm{d} u\left(\mathcal{O}_{S}(T S) \otimes \mathcal{O}_{S}\left(z_{c}\right)\right) / \mathrm{d} u\left(\mathcal{O}_{S}(T S)\right)
$$

of $\mathcal{N}_{u}$. Also, in this case, we denote by $E_{u}^{\text {cusp }}$ the subsheaf

$$
\left\{v \in \mathcal{O}_{S}\left(E_{u}\right) \mid \nabla v_{\mid z_{c}} \in \operatorname{Im}\left(\nabla \mathrm{d} u_{\mid z_{c}}\right)\right\}
$$

of $E_{u}$ and by $\mathcal{N}_{u}^{\text {cusp }}$ the quotient sheaf

$$
\mathcal{O}_{S}\left(E_{u}^{\text {cusp }}\right) / \mathrm{d} u\left(\mathcal{O}_{S}(T S)\right)
$$

We hence obtain the exact sequence

$$
0 \rightarrow \mathcal{O}_{S}(T S) \longrightarrow \mathcal{O}_{S}\left(E_{u}^{\text {cusp }}\right) \longrightarrow \mathcal{N}_{u}^{\text {cusp }} \rightarrow 0 .
$$

Note that from the inclusion $\mathcal{O}_{S}\left(E_{u}^{\text {cusp }}\right) \subset \mathcal{O}_{S}\left(E_{u}\right)$ follows the inclusion $\mathcal{N}_{u}^{\text {cusp }} \subset \mathcal{N}_{u}^{z_{c}} \oplus \mathbb{C}_{z_{c}}$.

Finally, denote by $\mathcal{O}_{S}\left(T S_{-\underline{z}}\right)\left(\operatorname{resp} . \mathcal{O}_{S}\left(E_{u,-\underline{z}}\right), \mathcal{O}_{S}\left(E_{u,-\underline{z}}^{\text {cusp }}\right), \mathcal{N}_{u,-\underline{z}}, \mathcal{N}_{u,-\underline{z}}^{z_{c}}\right.$, $\left.\mathcal{N}_{u,-\underline{z}}^{\text {cusp }}, \mathbb{C}_{z_{c},-\underline{z}}\right)$ the subsheaf of sections of $\mathcal{O}_{S}(T S)\left(\operatorname{resp} . \mathcal{O}_{S}\left(E_{u}\right), \mathcal{O}_{S}\left(E_{u}^{\text {cusp }}\right)\right.$, $\left.\mathcal{N}_{u}, \overline{\mathcal{N}}_{u}^{z_{c}}, \mathcal{N}_{u}^{\text {cusp }}, \mathbb{C}_{z_{c}}\right)$ which vanish at $\underline{z}$.

Proposition 1.2. - Let $\left(\left(u, J_{S}, J, \underline{x}\right), z_{c}\right) \in \mathcal{P}_{\text {cusp }}^{*} \backslash \mathcal{P}_{\text {hocusp }}^{*}$ Then, we have the inclusion $\mathbb{C}_{z_{c},-\underline{z}} \subset \mathcal{N}_{u,-\underline{z}}^{\text {cusp }}$ if and only if $z_{c} \in \underline{z}$ or $z_{c}$ is a degenerated cuspidal point of $u$. In both cases, $\nabla$ induces at $z_{c}$ a derivation $\nabla^{z_{c}}$ of sections of $\mathcal{N}_{u,-\underline{z}}^{z_{c}}$ such that the image of $\mathcal{N}_{u,-\underline{z}}^{\text {cusp }}$ in $\mathcal{N}_{u,-\underline{z}}^{z_{c}}$ under the projection $\mathcal{N}_{u,-\underline{z}} \rightarrow \mathcal{N}_{u,-\underline{z}}^{z_{c}-\underline{z}}$ with kernel $\mathbb{C}_{z_{c},-\underline{z}}$ is the subsheaf $\left\{v \in \mathcal{N}_{u,-\underline{z}}^{z_{c}} \mid \nabla^{z_{c}} v=0\right\}$.

Note that if $\left(\left(u, J_{S}, J, \underline{x}\right), z_{c}\right) \in \mathcal{P}_{\text {cusp }}^{*} \backslash \mathcal{P}_{\text {hocusp }}^{*}$ does not satisfy $\mathbb{C}_{z_{c},-\underline{z}} \subset$ $\mathcal{N}_{u,-\underline{z}}^{\text {cusp }}$, then the projection $\mathcal{N}_{u,-\underline{z}} \rightarrow \mathcal{N}_{u,-\underline{z}}^{z_{c}}$ with kernel $\mathbb{C}_{z_{c},-\underline{z}}$ establishes an isomorphism between the sheaves $\mathcal{N}_{u,-\underline{z}}^{\text {cusp }}$ and $\mathcal{N}_{u,-\underline{z}}^{z_{c}}$.

BULletin DE LA SOCiÉtÉ MATHÉMATIQUE DE FRANCE 
Proof. - There exist a complex coordinate $z$ of $\left(S, J_{S}\right)$ in a neighbourhood $U$ of $z_{c}$ as well as a local chart of $X$ in a neighbourhood of $u\left(z_{c}\right)$ such that the map $u$ writes $z \in U \mapsto\left(\left(z-z_{c}\right)^{2}, a\left(z-z_{c}\right)^{3}\right)+\left(z-z_{c}\right)^{3} \epsilon_{1}\left(z-z_{c}\right) \in \mathbb{C}^{2}$, where $a \in \mathbb{C}$ and $\epsilon_{1} \in L^{k, p}\left(U, \mathbb{C}^{2}\right), \epsilon_{1}\left(z_{c}\right)=0$ (see [8], Proposition 3). We can assume that the connection $\nabla$ is the standard connection given by this chart. Then, the image $\operatorname{Im}\left(\nabla \mathrm{d} u_{z_{c}}\right)$ is carried by the first coordinate axis of $\mathbb{C}^{2}$. Now, if $z_{c} \notin \underline{z}$ (resp. $\left.z_{c} \in \underline{z}\right)$, a local section of $\mathbb{C}_{z_{c},-\underline{z}}$ writes

$$
v_{z_{c}}=\mathrm{d} u\left(\frac{1}{z-z_{c}}\right)=\left(2,3 a\left(z-z_{c}\right)\right)+\left(z-z_{c}\right) \epsilon_{2}\left(z-z_{c}\right)
$$

(resp. $\left.v_{z_{c}}=\mathrm{d} u(1)=\left(2\left(z-z_{c}\right), 3 a\left(z-z_{c}\right)^{2}\right)+\left(z-z_{c}\right)^{2} \epsilon_{2}\left(z-z_{c}\right)\right)$, where $\epsilon_{2} \in L^{k-1, p}\left(U, \mathbb{C}^{2}\right), \epsilon_{2}\left(z_{c}\right)=0$. We deduce that $\left.\nabla v_{z_{c}}\right|_{z_{c}} \in \operatorname{Im}\left(\nabla \mathrm{d} u_{z_{c}}\right)$ if and only if $a=0$ or $z_{c} \in \underline{z}$. The first part of the proposition is proved. In both cases, the evaluation of $\nabla v_{z_{c}}$ at the point $z_{c}$ vanishes in $\mathcal{N}_{u,-\underline{z}}^{z_{c}}$. Thus, for every local section $v$ of $\mathcal{N}_{u,-\underline{z}}^{z_{c}}$, the evaluation of $\nabla v$ at the point $z_{c}$ does not depend on the choice of a lift of $v$ in $\mathcal{O}_{S}\left(E_{u,-\underline{z}}\right)$. We denote by

$$
\nabla^{z_{c}} v \in T_{u\left(z_{c}\right)} X / \operatorname{Im}\left(\nabla \mathrm{d} u_{z_{c}}\right)
$$

this value. A section $v$ of $\mathcal{O}_{S}\left(E_{u,-\underline{z}}\right)$ satisfies then $\nabla v_{\mid z_{c}} \in \operatorname{Im}\left(\nabla \mathrm{d} u_{\mid z_{c}}\right)$ if and only if the quotient section satisfies $\nabla^{z_{c}} v=0$.

Remember that the operator

$$
D: L^{k, p}\left(S, E_{u,-\underline{z}}^{\text {cusp }}\right) \longrightarrow L^{k-1, p}\left(S, \Lambda^{0,1} S \otimes E_{u}^{\text {cusp }}\right)
$$

induces a quotient operator

$\bar{D}: L^{k, p}\left(S, \mathcal{N}_{u,-\underline{z}}^{\text {cusp }}\right):=L^{k, p}\left(S, E_{u,-\underline{z}}^{\text {cusp }}\right) / \mathrm{d} u\left(L^{k, p}\left(S, T S_{-\underline{z}}\right) \rightarrow L^{k-1, p}\left(S, \Lambda^{0,1} S \otimes N_{u}\right)\right.$.

Here, $N_{u}$ denotes the normal bundle of $u$ and $N_{u,-\underline{z}}=N_{u} \otimes \mathcal{O}_{S}(-\underline{z})$. From the short exact sequence of complexes

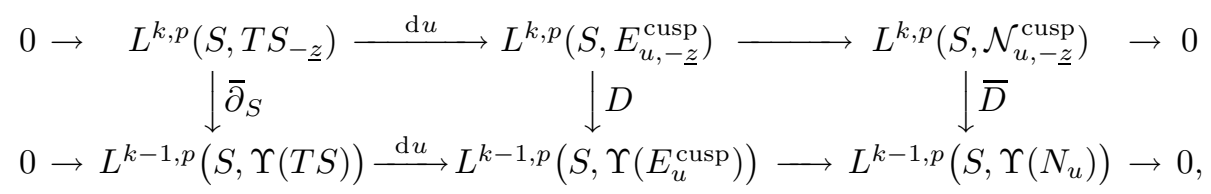

where $\Upsilon(X)=\Lambda^{0,1} S \otimes X$, we deduce the long exact sequence

$$
\begin{aligned}
0 \rightarrow H^{0}\left(S, T S_{-\underline{z}}\right) & \longrightarrow H_{D}^{0}\left(S, E_{u,-\underline{z}}^{\text {cusp }}\right) \longrightarrow H_{\bar{D}}^{0}\left(S, \mathcal{N}_{u,-\underline{z}}^{\text {cusp }}\right) \\
& \longrightarrow H^{1}\left(S, T S_{-\underline{z}}\right) \longrightarrow H_{D}^{1}\left(S, E_{u,-\underline{z}}^{\text {cusp }}\right) \longrightarrow H_{\bar{D}}^{1}\left(S, \mathcal{N}_{u,-\underline{z}}^{\text {cusp }}\right) \rightarrow 0
\end{aligned}
$$

where $H_{D}^{0}, H_{\bar{D}}^{0}$ (resp. $H_{D}^{1}, H_{\bar{D}}^{1}$ ) denote the kernels (resp. cokernels) of the operators $D, \bar{D}$ on the associated sheaves. In particular,

$$
\begin{aligned}
& \operatorname{ind}_{\mathbb{R}}(\bar{D})=\operatorname{ind}_{\mathbb{R}}(D)-\operatorname{ind}_{\mathbb{R}}\left(\bar{\partial}_{S}\right)=2\left(c_{1}(X) d+1-2 \# \underline{z}\right)-2(3-\# \underline{z})=0 . \\
& \text { томе } 134-2006-\mathrm{N}^{\mathrm{o}} 2
\end{aligned}
$$




\subsection{Moduli space of real rational cuspidal pseudo-holomorphic curves}

1.3.1. Gauge action of $\mathcal{D} \operatorname{iff}(S, \underline{z})$ on $\mathcal{P}_{\text {cusp }}^{*}$ - Denote by $\operatorname{Diff}(S, z)$ the group of diffeomorphisms of class $C^{\ell+1}$ of $S$, which either preserve the orientation and fix $\underline{z}$, or reverse the orientation and induce the permutation on $\underline{z}$ associated to $\tau$. Let $\operatorname{Diff}^{+}(S, z)$ (resp. $\left.\mathcal{D}_{\text {iff }}^{-}(S, z)\right)$ be the subgroup of $\mathcal{D}$ iff $(S, z)$ made of orientation preserving diffeomorphisms (resp. its complement in $\mathcal{D}$ iff $(S, z)$ ). Let $s_{*}$ be the morphism $\mathcal{D}$ iff $(S, z) \rightarrow \mathbb{Z} / 2 \mathbb{Z}$ having kernel $\mathcal{D}_{\text {iff }}^{+}(S, z)$. The group $\operatorname{Diff}(S, z)$ acts on the pair $\left(\mathcal{P}_{\text {cusp }}^{*}, \mathcal{P}_{\text {hocusp }}^{*}\right)$ by

$$
\phi .\left(\left(u, J_{S}, J, \underline{x}\right), z_{c}\right)=\left\{\begin{array}{rr}
\left(\left(u \circ \phi^{-1},\left(\phi^{-1}\right)^{*} J_{S}, J, \underline{x}\right), \phi\left(z_{c}\right)\right) & \text { if } s_{*}(\phi)=+1, \\
\left(\left(c_{X} \circ u \circ \phi^{-1},\left(\phi^{-1}\right)^{*} J_{S}, \overline{c_{X}}{ }^{*}(J), c_{X}(\underline{x})\right), \phi\left(z_{c}\right)\right) & \text { if } s_{*}(\phi)=-1,
\end{array}\right.
$$

where

$$
\left(\phi^{-1}\right)^{*} J_{S}=s_{*}(\phi) \mathrm{d} \phi \circ J_{S} \circ \mathrm{d} \phi^{-1} \quad \text { and } \quad{\overline{c_{X}}}^{*}(J)=-\mathrm{d} c_{X} \circ J \circ \mathrm{d} c_{X} .
$$

With the exception of the identity, only the order 2 elements of $\mathcal{D i f f}^{-}(S, z)$ may have non empty fixed point set in $\mathcal{P}_{\text {cusp }}^{*}$. In particular, two such involutions have disjoint fixed point sets (compare [11], Lemma 1.3). Moreover, the operators $D$ and $\bar{D}$ are $\operatorname{Diff}(S, z)$ equivariants (compare [11], Lemma 1.5). Now, if $\left(\left(u, J_{S}, J, \underline{x}\right), z_{c}\right)$ is fixed by some order 2 element $c_{S}$ of $\mathcal{D i f f}^{-}(S, z)$, we denote by

$$
H_{D}^{0}\left(S, E_{u,-\underline{z}}^{\text {cusp }}\right)_{ \pm 1}, \quad H_{D}^{1}\left(S, E_{u,-\underline{z}}^{\text {cusp }}\right)_{ \pm 1}
$$

(resp. $\left.H_{D}^{0}\left(S, \mathcal{N}_{u,-\underline{z}}^{\text {cusp }}\right)_{ \pm 1}, H_{D}^{1}\left(S, \mathcal{N}_{u,-\underline{z}}^{\text {cusp }}\right)_{ \pm 1}\right)$ the eigenspaces associated to the eigenvalue \pm 1 of the action of $c_{S}$ on the kernel and the cokernel of $D$ (resp. of $\bar{D})$.

1.3.2. Moduli spaces $\mathcal{M}_{\text {cusp }}^{d}, \mathbb{R} \mathcal{M}_{\text {cusp }}^{d}$ and the projections $\pi, \pi_{\mathbb{R}}$. - Denote by

$$
\mathcal{M}_{\text {cusp }}^{d} \quad\left(\text { resp. } \mathcal{M}_{\text {hocusp }}^{d}\right)
$$

the quotient of $\mathcal{P}_{\text {cusp }}^{*}$ (resp. $\quad \mathcal{P}_{\text {hocusp }}^{*}$ ) by the action of $\operatorname{Diff}^{+}(S, z)$. This space $\mathcal{M}_{\text {cusp }}^{d}$ is the moduli space of rational pseudo-holomorphic curves which realize the homology class $d$ and are not immersed, whereas $\mathcal{M}_{\text {hocusp }}^{d}$ is the moduli space of such curves which have a cuspidal point of order greater than 1 , that is of the form

$$
z \longmapsto\left(z^{a}, z^{b}+o\left(z^{b}\right)\right) \text { with } 2<a<b .
$$

The projection

$$
\pi:\left(\left(u, J_{S}, J, \underline{x}\right), z_{c}\right) \in \mathcal{P}_{\text {cusp }}^{*} \longmapsto(J, \underline{x}) \in \mathcal{J}_{\omega} \times X^{\nu}
$$

induces on the quotient a projection $\mathcal{M}_{\text {cusp }}^{d} \rightarrow \mathcal{J}_{\omega} \times X^{\nu}$ still denoted by $\pi$.

BULletin DE LA SOCIÉtÉ MATHÉMATIQUE DE FRANCE 
Proposition 1.3. - The space $\mathcal{M}_{\text {cusp }}^{d}$ is a separable Banach manifold of class $C^{\ell-k}$, and $\pi$ is Fredholm of vanishing index. Moreover, the space $\mathcal{M}_{\text {hocusp }}^{d}$ is a separable Banach submanifold of class $C^{\ell-k}$ and complex codimension 2 of $\mathcal{M}_{\text {cusp. }}^{d}$ Finally, if $\left[\left(u, J_{S}, J, \underline{x}\right), z_{c}\right] \in \mathcal{M}_{\text {cusp }}^{d} \backslash \mathcal{M}_{\text {hocusp }}^{d}$, then we have the isomorphisms

$$
\begin{gathered}
\operatorname{ker} \mathrm{d} \pi_{\mid\left(\left(u, J_{S}, J, \underline{x}\right), z_{c}\right)} \cong H_{D}^{0}\left(S, \mathcal{N}_{u,-\underline{z}}^{\text {cusp }}\right), \\
\operatorname{coker} \mathrm{d} \pi_{\mid\left(\left(u, J_{S}, J, \underline{x}\right), z_{c}\right)} \cong H_{D}^{1}\left(S, \mathcal{N}_{u,-\underline{z}}^{\text {cusp }}\right) .
\end{gathered}
$$

Proof. — The proof is analogous to the one of Corollary 2.2.3 of [6]. The action of $\mathcal{D i f f}^{+}(S, z)$ on $\mathcal{P}_{\text {cusp }}^{*}$ and $\mathcal{P}_{\text {hocusp }}^{*}$ is smooth, fixed point free and admits a closed supplement. From Proposition 1.1 thus follows that $\mathcal{M}_{\text {cusp }}^{d}$ and $\mathcal{M}_{\text {hocusp }}^{d}$ are separable Banach manifolds, the latter being of codimension 4 in the former. Moreover, since $\nabla \mathrm{d} u_{\left.\right|_{z_{c}}} \neq 0$, we have

$$
\begin{aligned}
\operatorname{ker} \mathrm{d} \pi_{\mid\left[\left(u, J_{S}, J, \underline{x}\right), z_{c}\right]}=\left\{\left(v, \dot{J}_{S}, 0,0, \dot{z}_{c}\right) \in T_{\left(u, J_{S}, J, \underline{x}\right.} \mathcal{P}^{*} \times T_{z_{c}} S\right. \\
\left.\mid \nabla v v_{z_{c}}+\nabla_{\dot{z}_{c}} \mathrm{~d} u=0\right\} / T_{\mathrm{Id}} \mathcal{D}_{i f f}^{+}(S, z) \\
=\left\{\left(v, \dot{J}_{S}\right) \in L^{k, p}\left(S, E_{u,-\underline{z}}^{\text {cusp }}\right) \times T_{J_{S}} \mathcal{J}_{S}\right. \\
\left.\quad \mid D v=-J \circ \mathrm{d} u \circ \dot{J}_{S}\right\} / T_{\mathrm{Id}} \mathcal{D i f f}^{+}(S, z) \\
=\left\{v \in L^{k, p}\left(S, E_{u,-\underline{z}}^{\text {cusp }}\right) \mid \exists \phi \in L^{k-1, p}\left(S, \Lambda^{0,1} S \otimes T S\right),\right. \\
D v=\mathrm{d} u(\phi)\} / \mathrm{d} u\left(L^{k, p}\left(S, T S_{-\underline{z}}\right)\right) \\
=H_{D}^{0}\left(S, \mathcal{N}_{u,-\underline{z}}^{\text {cusp }}\right),
\end{aligned}
$$

from the long exact sequence given at the end of $\S 1.2$. Likewise,

$$
\begin{aligned}
\operatorname{Im} \mathrm{d} & \pi_{\mid}\left[\left(u, J_{S}, J, \underline{x}\right), z_{c}\right] \\
=\{ & (\dot{J}, \underline{\dot{x}}) \in T_{J} \mathcal{J}_{\omega} \times T_{\underline{x}} X^{\nu} \\
& \mid \exists\left(v, \dot{J}_{S}, \dot{z}_{c}\right) \in L^{k, p}\left(S, E_{u,-\underline{z}}\right) \times T_{J_{S}} \mathcal{J}_{S} \times T_{z_{c}} S, \\
& \left.\quad D v+J \circ \mathrm{d} u \circ \dot{J}_{S}=-\dot{J} \circ \mathrm{d} u \circ J_{S}, \nabla v_{z_{c}}+\nabla_{\dot{z}_{c}} \mathrm{~d} u=0, v(\underline{z})=\underline{\dot{x}}\right\}
\end{aligned}
$$

so that

$$
\text { coker } \mathrm{d} \pi_{\mid\left[\left(u, J_{S}, J, \underline{x}\right), z_{c}\right]}=L^{k-1, p}\left(S, \Lambda^{0,1} S \otimes E_{u}\right) \times T_{\underline{x}} X^{c_{1}(X) d-2} / \operatorname{Im}(\widehat{\mathrm{D}} \times \mathrm{ev}),
$$

where

$$
\begin{aligned}
\widehat{D}:\left(v, \dot{J}_{S}\right) \in L^{k, p}\left(S, E_{u}^{\text {cusp }}\right) & \times T_{J_{S}} \mathcal{J}_{S} \\
& \longmapsto D v+J \circ \mathrm{d} u \circ \dot{J}_{S} \in L^{k-1, p}\left(S, \Lambda^{0,1} S \otimes E_{u}\right), \\
\mathrm{ev}: v \in L^{k, p}\left(S, E_{u}^{\text {cusp }}\right) & \longmapsto v(\underline{z}) \in T_{\underline{x}} X^{c_{1}(X) d-2} .
\end{aligned}
$$

By definition, coker $D=H_{D}^{1}\left(S, E_{u}^{\text {cusp }}\right)$. From the short exact sequence

$$
0 \rightarrow E_{u,-\underline{z}}^{\text {cusp }} \longrightarrow E_{u}^{\text {cusp }} \stackrel{\text { ev }}{\longrightarrow} T_{\underline{x}} X^{c_{1}(X) d-2} \rightarrow 0
$$

TOME $134-2006-\mathrm{N}^{\mathrm{O}} 2$ 
we deduce the long exact sequence

$$
\begin{aligned}
\cdots \longrightarrow H_{D}^{0}\left(S, E_{u}^{\text {cusp }}\right) & \longrightarrow H^{0}\left(S, T_{\underline{x}} X^{c_{1}(X) d-2}\right) \\
& \longrightarrow H_{D}^{1}\left(S, E_{u,-\underline{z}}^{\text {cusp }}\right) \longrightarrow H_{D}^{1}\left(S, E_{u}^{\text {cusp }}\right) \rightarrow 0 .
\end{aligned}
$$

Hence, the cokernel of $D \times$ ev in $L^{k-1, p}\left(S, \Lambda^{0,1} S \otimes E_{u}\right) \times T_{\underline{x}} X^{c_{1}(X) d-2}$ is isomorphic to $H_{D}^{1}\left(S, E_{u,-\underline{z}}^{\text {cusp }}\right)$. From the long exact sequence given at the end of $\S 1.2$, we deduce that the cokernel of $\widehat{D} \times$ ev and hence the one of $\mathrm{d} \pi_{\mid\left[\left(u, J_{S}, J, \underline{x}\right), z_{c}\right]}$ is isomorphic to $H_{D}^{1}\left(S, \mathcal{N}_{u,-\underline{z}}^{\text {cusp }}\right)$.

The manifolds $\mathcal{M}_{\text {cusp }}^{d}$ and $\mathcal{M}_{\text {hocusp }}^{d}$ are equipped with an action of the group

$$
\operatorname{Diff}(S, z) / \mathcal{D i f f}^{+}(S, z) \cong \mathbb{Z} / 2 \mathbb{Z} \text {. }
$$

We denote by $\mathbb{R} \mathcal{M}_{\text {cusp }}^{d}$ and $\mathbb{R} \mathcal{M}_{\text {hocusp }}^{d}$ the fixed point sets of these actions.

This space $\mathbb{R} \mathcal{M}_{\text {cusp }}^{d}$ is the moduli space of real rational pseudo-holomorphic curves which realize the homology class $d$ and have a real cuspidal point, whereas $\mathbb{R} \mathcal{M}_{\text {hocusp }}^{d}$ is the moduli space of such real curves which have a real cuspidal point of order greater than one, that is of the form

$$
z \longmapsto\left(z^{a}, z^{b}+o\left(z^{b}\right)\right) \quad \text { with } 2<a<b .
$$

The projection $\pi$ is then $\mathbb{Z} / 2 \mathbb{Z}$-equivariant as soon as $\mathcal{J}_{\omega} \times X^{c_{1}(X) d-2}$ is equipped with the action $\overline{c_{X}}{ }^{*} \times c_{\tau}$, where

$$
{\overline{c_{X}}}^{*}: J \in \mathcal{J}_{\omega} \longmapsto-\mathrm{d} c_{X} \circ J \circ \mathrm{d} c_{X} \in \mathcal{J}_{\omega}
$$

and $c_{\tau}$ has been defined at the beginning of $\S 1$. Denote by $\pi_{\mathbb{R}}$ the induced projection

$$
\mathbb{R} \mathcal{M}_{\text {cusp }}^{d} \longrightarrow \mathbb{R} \mathcal{J}_{\omega} \times \mathbb{R}_{\tau} X^{c_{1}(X) d-2} .
$$

Proposition 1.4. - The spaces $\mathbb{R} \mathcal{M}_{\text {cusp }}^{d}$ and $\mathbb{R} \mathcal{M}_{\text {hocusp }}^{d}$ are separable Banach manifolds of class $C^{\ell-k}$, the latter being of codimension 2 in the former. Moreover, $\pi_{\mathbb{R}}$ is Fredholm of vanishing index. Finally, if $\left[\left(u, J_{S}, J, \underline{x}\right), z_{c}\right]$ belongs to $\mathbb{R} \mathcal{M}_{\text {cusp }}^{d} \backslash \mathbb{R} \mathcal{M}_{\text {hocusp }}^{d}$, then we have the isomorphisms

$$
\begin{aligned}
& \operatorname{ker} \mathrm{d} \pi_{\mathbb{R}} \mid\left(\left(u, J_{S}, J, \underline{x}\right), z_{c}\right) \\
& \operatorname{coker} \mathrm{d} \pi_{\mathbb{R} \mid\left(\left(u, J_{S}, J, \underline{x}\right), z_{c}\right)} \cong H_{D}^{0}\left(S, \mathcal{N}_{u,-\underline{z}}^{\text {cusp }}\right)_{+1}, \\
& \left.\cos , \mathcal{N}_{u,-\underline{z}}^{\text {cusp }}\right)_{+1} .
\end{aligned}
$$

\subsection{Critical points of $\pi_{\mathbb{R}}$}

Lemma 1.5. - The point $\left[\left(u, J_{S}, J, \underline{x}\right), z_{c}\right] \in \mathbb{R} \mathcal{M}_{\text {cusp }}^{d} \backslash \mathbb{R} \mathcal{M}_{\text {hocusp }}^{d}$ is critical for $\pi_{\mathbb{R}}$ if and only if one of the following:

1) the differential $\mathrm{d} u$ vanishes outside $z_{c}$;

2) the cuspidal point $z_{c}$ is degenerated;

3) one has $z_{c} \in \underline{z}$.

BULLETIN DE LA SOCIÉtÉ MATHÉMATIQUE DE FRANCE 
Proof. - From Proposition $1.4,\left[\left(u, J_{S}, J, \underline{x}\right), z_{c}\right]$ is a critical point of $\pi_{\mathbb{R}}$ if and only if

$$
H_{D}^{0}\left(S, \mathcal{N}_{u,-\underline{z}}^{\text {cusp }}\right)_{+1} \cong H_{D}^{1}\left(S, \mathcal{N}_{u,-\underline{z}}^{\text {cusp }}\right)_{+1} \neq\{0\} .
$$

We deduce from Proposition 1.2 that 2) and 3) are indeed critical points of $\pi_{\mathbb{R}}$, since in these cases $\mathbb{C}_{z_{c},-\underline{z}} \subset \mathcal{N}_{u,-\underline{z}}^{\text {cusp }}$ and

$$
\{0\} \neq H^{0}\left(S, \mathbb{C}_{z_{c},-\underline{z}}\right)_{+1} \subset H_{D}^{0}\left(S, \mathcal{N}_{u,-\underline{z}}^{\text {cusp }}\right)_{+1} .
$$

In the same way, if $z_{c}$ is a real ordinary cusp of $u$ distinct from $\underline{z}$, then from Proposition 1.2, $\mathcal{N}_{u,-\underline{z}}^{\text {cusp }}=\mathcal{N}_{u,-\underline{z}}^{z_{c}}$. If d $u$ does not vanish outside $z_{c}$, then $\mathcal{N}_{u,-\underline{z}}^{z_{c}} \cong$ $\mathcal{O}_{S}\left(N_{u,-\underline{z}}\right)$ so that $H_{D}^{1}\left(S, \mathcal{N}_{u,-\underline{z}}^{\text {cusp }}\right)_{+1}=\{0\}$. Otherwise, the sheaf $\mathcal{N}_{u,-\underline{z}}^{\text {cusp }}$ carries some skyscraper part and $H_{D}^{0}\left(S, \mathcal{N}_{u,-\underline{z}}^{\text {cusp }}\right)_{+1} \neq\{0\}$, hence the result.

A detailed study of critical points of type 2) will be carried out in $\S 2$. In particular, we will prove in Lemma 2.4 that if $z$ is a local coordinate in a neighbourhood of $z_{c}$ which is adapted to $u$, that is for which the order 3 jet of $u$ reads $j_{2}\left(z_{c}\right)\left(z-z_{c}\right)^{2}+o\left(\left|z-z_{c}\right|^{3}\right)$, then the homogeneous part of order 5 of its jet is a complex monomial denoted by $j_{5}\left(z_{c}\right)\left(z-z_{c}\right)^{5}$. Also, note that if $u$ has two distinct ordinary cusps, then

$$
H_{D}^{1}\left(S, \mathcal{N}_{u,-\underline{z}}^{\text {cusp }}\right)_{+1} \cong H_{D}^{1}\left(S, \mathcal{N}_{u,-\underline{z}}^{z_{c}}\right)_{+1}
$$

is of dimension 1 and from Riemann-Roch duality, it is isomorphic to (see [11], Lemma 1.7)

$$
H_{D^{*}}^{0}\left(S, K_{S} \otimes \mathcal{N}_{u,-\underline{z}}^{z_{c}}\right)_{-1} .
$$

Finally, remember that a stratum of codimension $m \in \mathbb{N}$ of a separable Banach manifold $N$ is by definition the image of a separable Banach manifold $M$ under a smooth Fredholm map $f$ of Fredholm index $-m$ such that all the limits of sequences $\phi\left(x_{n}\right)$, where $x_{n}$ is a diverging sequence in $M$, belong to a countable union of strata of higher codimensions.

LEMMA 1.6. - 1) The set of points $\left[\left(u, J_{S}, J, \underline{x}\right), z_{c}\right] \in \mathbb{R} \mathcal{M}_{\text {cusp }}^{d}$ for which $u$ has two distinct real ordinary cusps and ordinary double points as singularities, all of which being outside $\underline{x}$ and for which any generator $\psi$ of

$$
H_{D^{*}}^{0}\left(S, K_{S} \otimes \mathcal{N}_{u,-\underline{z}}^{z_{c}}\right)_{-1}=H_{D}^{1}\left(S, \mathcal{N}_{u,-\underline{z}}^{\text {cusp }}\right)_{+1}^{*}
$$

does not vanish at the cusps, is a stratum of codimension 1 of $\mathbb{R} \mathcal{M}_{\text {cusp }}^{d}$.

2) The set of points $\left[\left(u, J_{S}, J, \underline{x}\right), z_{c}\right] \in \mathbb{R} \mathcal{M}_{\text {cusp }}^{d}$ for which $u$ has a degenerated cusp and transversal double points as singularities, all of which being outside $\underline{x}$, and for which $j_{5}\left(z_{c}\right)$ is not colinear to $j_{2}\left(z_{c}\right)$ in an adapted coordinate, is a stratum of codimension 1 of $\mathbb{R} \mathcal{M}_{\text {cusp }}^{d}$

3) The set of points $\left[\left(u, J_{S}, J, \underline{x}\right), z_{c}\right] \in \mathbb{R} \mathcal{M}_{\text {cusp }}^{d}$ for which $u$ has a real ordinary cusp at $z_{c} \in \underline{z}$ and only ordinary double points as other singularities, all of which being outside $\underline{x}$, is a stratum of codimension 1 of $\mathbb{R} \mathcal{M}_{\text {cusp }}^{d}$.

TOME $134-2006-\mathrm{N}^{\mathrm{O}} 2$ 
The union of all critical points of $\pi_{\mathbb{R}}$ not listed above belong to a countable union of strata of codimension at least 2 in $\mathbb{R} \mathcal{M}_{\text {cusp }}^{d}$.

Proof. - The case 2 follows from Proposition 2.6. All the other cases can be proved in the same way as Propositions 2.7 and 2.8 of [11]. For the sake of concision, these proofs are not reproduced here.

REMARK 1.7. - There are actually only finitely many strata occuring in Lemma 1.6, since a pseudo-holomorphic curve which realize the given homology class $d$ may have only finitely many different types of singularities. Moreover, as soon as $k$ and $\ell$ are large enough, all these strata are images of Banach manifolds of class $C^{2}$ at least. We will use only the following fact: a generic path $\gamma:[0,1] \rightarrow \mathbb{R} \mathcal{J}_{\omega} \times \mathbb{R}_{\tau} X^{c_{1}(X) d-2}$ avoids the image under $\pi_{\mathbb{R}}$ of every stratum of codimension at least 2 .

The critical points of $\pi_{\mathbb{R}}$ listed in Lemma 1.6 are said to be generic.

ThEOREM 1.8. - The generic critical points of $\pi_{\mathbb{R}}$ are non degenerated.

Proof. - In the case of critical points of type 1 given by Lemmas 1.5 and 1.6, the proof is readily the same as the one of Lemma 2.13 of [11]; it is not reproduced here. Let $\left[\left(u, J_{S}, J, \underline{x}\right), z_{c}\right] \in \mathbb{R} \mathcal{M}_{\text {cusp }}^{d}$ be a generic critical point of type 2 or 3 given by Lemma 1.6. We have to prove that the quadratic form

$$
\begin{aligned}
\nabla \mathrm{d} \pi_{\mathbb{R} \mid\left[\left(u, J_{S}, J, \underline{x}\right), z_{c}\right]}: \operatorname{ker} \mathrm{d} \pi_{\mathbb{R} \mid\left[\left(u, J_{S}, J, \underline{x}\right), z_{c}\right]} \times \operatorname{ker} \mathrm{d} \pi_{\mathbb{R} \mid\left[\left(u, J_{S}, J, \underline{x}\right), z_{c}\right]} & \longrightarrow \operatorname{coker} \mathrm{d} \pi_{\mathbb{R} \mid\left[\left(u, J_{S}, J, \underline{x}\right), z_{c}\right]}
\end{aligned}
$$

is non degenerated. Write $\pi_{\mathbb{R}}=\left(\pi_{\mathbb{R}}^{1}, \pi_{\mathbb{R}}^{2}\right), \mathbb{R} \mathcal{M}_{\text {cusp }}^{d}(\underline{x})=\left(\pi_{\mathbb{R}}^{2}\right)^{-1}(\underline{x})$ and

$$
\pi_{\mathbb{R}}^{J}:\left[\left(u, J_{S}, J, \underline{x}\right), z_{c}\right] \in \mathbb{R} \mathcal{M}_{\text {cusp }}^{d}(\underline{x}) \longmapsto J \in \mathbb{R} \mathcal{J}_{\omega}
$$

the restriction of $\pi_{\mathbb{R}}^{1}$ to $\mathbb{R} \mathcal{M}_{\text {cusp }}^{d}(\underline{x})$. The quadratic forms $\nabla \mathrm{d} \pi_{\mathbb{R} \mid\left[\left(u, J_{S}, J, \underline{x}\right), z_{c}\right]}$ and $\nabla \mathrm{d} \pi_{\mathbb{R}}^{J} \mathbf{I}\left[\left(u, J_{S}, J\right), z_{c}\right]$ are of the same nature. Moreover, the kernel and cokernel of the map $\mathrm{d} \pi_{\mathbb{R}}^{J}$ are the same as the ones of the operator

$$
\begin{aligned}
-\widehat{D}_{\mathbb{R}}:\left(v, \dot{J}_{S}, \dot{J}, \dot{z}_{c}\right) \in T_{\left[\left(u, J_{S}, J\right), z_{c}\right]} & \mathbb{R} \mathcal{M}_{\text {cusp }}^{d}(\underline{x}) \\
& \longmapsto \dot{J} \circ \mathrm{d} u \circ J_{S} \in L^{k-1, p}\left(S, \Lambda^{0,1} S \otimes N_{u}\right) .
\end{aligned}
$$

From the relation $D v+J \circ \mathrm{d} u \circ \dot{J}_{S}+\dot{J} \circ \mathrm{d} u \circ J_{S}=0$, we deduce that

$$
\widehat{D}_{\mathbb{R}}\left(v, \dot{J}_{S}, \dot{J}, \dot{z}_{c}\right)=D v+J \circ \mathrm{d} u \circ \dot{J}_{S} .
$$

We then have to prove that

$$
\nabla \widehat{D}_{\mathbb{R} \mid\left[\left(u, J_{S}, J\right), z_{c}\right]}: H_{D}^{0}\left(S, \mathcal{N}_{u,-\underline{z}}^{\text {cusp }}\right)_{+1}^{2} \longrightarrow H_{D}^{1}\left(S, \mathcal{N}_{u,-\underline{z}}^{\text {cusp }}\right)_{+1}
$$

is non degenerated. Let $\left(v_{1}, \dot{J}_{S}^{1}, 0, \dot{z}_{c}^{1}\right)$ be a generator of $H_{D}^{0}\left(S, \mathcal{N}_{u,-z}^{\text {cusp }}\right)_{+1}$, then from Proposition 1.2, $v_{1}=\mathrm{d} u\left(\tilde{v}_{1}\right)$ where $\tilde{v}_{1} \in L^{k, p}\left(S, T S_{-\underline{z}} \otimes \mathcal{O}_{S}\left(z_{c}\right)\right)_{+1}$, that is $\tilde{v}_{1}$ is a meromorphic vector field on $S$ either having a simple pole at $z_{c}$ if $z_{c} \notin \underline{z}$ 
or which does not vanish at $z_{c}$ otherwise. In the same way, let $\left(v_{2}, \dot{J}_{S}^{2}, 0, \dot{z}_{c}^{2}\right) \in$ $T_{\left[u, J_{S}, J, z_{c}\right]} \mathbb{R} \mathcal{M}_{\text {cusp }}^{d}(\underline{x})$, then

$$
\begin{aligned}
& \left(\nabla_{\left(v_{2}, \dot{J}_{S}^{2}, 0, \dot{z}_{c}^{2}\right)} \widehat{D}_{\mathbb{R}}\right)\left(v_{1}, \dot{J}_{S}^{1}, 0, \dot{z}_{c}^{1}\right) \\
& \quad=\left(\nabla_{\left(v_{2}, \dot{J}_{S}^{2}, 0, \dot{z}_{c}^{2}\right)} D_{\mathbb{R}}\right)\left(v_{1}\right)+\left(\nabla_{v_{2}} \mathrm{~d} u\right) \circ J_{S} \circ \dot{J}_{S}^{1} \bmod (\operatorname{Im}(\mathrm{d} u)) .
\end{aligned}
$$

Moreover, after differentiation of the relation $D \circ \mathrm{d} u=\mathrm{d} u \circ \bar{\partial}_{S}$, we deduce $\left(\nabla_{\left(v_{2}, \dot{J}_{S}^{2}, 0, \dot{z}_{c}^{2}\right)} D_{\mathbb{R}}\right)\left(\mathrm{d} u\left(\tilde{v}_{1}\right)\right)+D_{\mathbb{R}} \circ\left(\nabla_{v_{2}} \mathrm{~d} u\right)\left(\tilde{v}_{1}\right)=\nabla_{v_{2}} \mathrm{~d} u \circ \bar{\partial}_{S}\left(\tilde{v}_{1}\right) \bmod (\operatorname{Im}(\mathrm{d} u))$.

Since $0=D v_{1}+J \circ \mathrm{d} u \circ \dot{J}_{S}^{1}=\mathrm{d} u\left(\bar{\partial}_{S}\left(\tilde{v}_{1}\right)+J_{S} \dot{J}_{S}^{1}\right)$ forces $\bar{\partial}_{S}\left(\tilde{v}_{1}\right)+J_{S} \dot{J}_{S}^{1}=0$, we deduce

$$
\left(\nabla_{\left(v_{2}, \dot{J}_{S}^{2}, 0, \dot{z}_{c}^{2}\right)} \widehat{D}_{\mathbb{R}}\right)\left(v_{1}, \dot{J}_{S}^{1}, 0, \dot{z}_{c}^{1}\right)=-D_{\mathbb{R}}\left(\nabla_{v_{2}} \mathrm{~d} u\right)\left(\tilde{v}_{1}\right) \quad \bmod (\operatorname{Im}(\mathrm{d} u)) .
$$

We thus have to prove that the projection

$$
D_{\mathbb{R}}\left(\nabla_{\tilde{v}_{1}} \mathrm{~d} u\left(\tilde{v}_{1}\right)\right) \in H_{D}^{1}\left(S ; \mathcal{N}_{u,-\underline{z}}^{\text {cusp }}\right)_{+1} \cong \mathbb{R}
$$

does not vanish as soon as $\tilde{v}_{1} \neq 0$. Now the operator

$$
D_{\mathbb{R}}: L^{k, p}\left(S, \mathcal{N}_{u,-\underline{z}}\right)_{+1} \longrightarrow L^{k-1, p}\left(S, \Lambda^{0,1} \otimes N_{u}\right)_{+1}
$$

is an isomorphism, and $L^{k, p}\left(S, \mathcal{N}_{u,-\underline{z}}^{\text {cusp }}\right)_{+1}=\left\{v \in L^{k, p}\left(S, \mathcal{N}_{u,-\underline{z}}\right)_{+1} \mid \nabla^{z_{c}} v=0\right\}$ (see Proposition 1.2) is a closed hyperplane of $L^{k, p}\left(S, \mathcal{N}_{u,-\underline{z}}\right)_{+1}$. We hence have to prove that $v=\nabla_{\tilde{v}_{1}} \mathrm{~d} u\left(\tilde{v}_{1}\right)$ does not satisfy $\nabla^{z_{c}} v=0$.

The map $u$ can be written in an adapted local chart at $z_{c}$ as

$$
u(z)=j_{2}\left(z_{c}\right)\left(z-z_{c}\right)^{2}+\cdots+j_{5}\left(z_{c}\right)\left(z-z_{c}\right)^{5}+o\left(\left|z-z_{c}\right|^{5}\right),
$$

where $\left(j_{2}\left(z_{c}\right), j_{5}\left(z_{c}\right)\right)$ does form a basis of $\mathbb{R}^{2}$ if $z_{c} \notin \underline{z}$ or

$$
u(z)=j_{2}\left(z_{c}\right)\left(z-z_{c}\right)^{2}+j_{3}\left(z_{c}\right)\left(z-z_{c}\right)^{3}+o\left(\left|z-z_{c}\right|^{3}\right),
$$

where $\left(j_{2}\left(z_{c}\right), j_{3}\left(z_{c}\right)\right)$ does form a basis of $\mathbb{R}^{2}$ if $z_{c} \in \underline{z}$. We can assume that the connection $\nabla$ is the standard one given by this chart. Then in the first case, $\tilde{v}_{1}=1 /\left(z-z_{c}\right)$ and

$$
\begin{aligned}
v=\left(\nabla_{\tilde{v}_{1}} \mathrm{~d} u\right)\left(\tilde{v}_{1}\right) & =\frac{2 j_{2}\left(z_{c}\right)}{\left(z-z_{c}\right)^{2}}+20 j_{5}\left(z_{c}\right)\left(z-z_{c}\right)+o\left(\left|z-z_{c}\right|\right) \\
& =15 j_{5}\left(z_{c}\right)\left(z-z_{c}\right)+o\left(\left|z-z_{c}\right|\right) \bmod (\operatorname{Im}(\mathrm{d} u)) .
\end{aligned}
$$

And in the second case, $\tilde{v}_{1}=1$ and

$$
\begin{aligned}
v & =\left(\nabla_{\tilde{v}_{1}} \mathrm{~d} u\right)\left(\tilde{v}_{1}\right) \\
& =2 j_{2}\left(z_{c}\right)+6 j_{3}\left(z_{c}\right)\left(z-z_{c}\right)+o\left(\left|z-z_{c}\right|\right) .
\end{aligned}
$$

In the first case, $\nabla v_{z_{c}}=15 j_{5}\left(z_{c}\right) \mathrm{d} z$ while in the second case $\nabla v_{\mid z_{c}}=$ $6 j_{3}\left(z_{c}\right) \mathrm{d} z$. In both cases, the projection $\nabla^{z_{c}} v$ of $\nabla v_{z_{c}}$ in the normal bundle does not satisfy $\nabla^{z_{c}} v=0$, hence the result.

TOME $134-2006-\mathrm{N}^{\mathrm{O}} 2$ 
1.5. Gromov compactification $\mathbb{R} \overline{\mathcal{M}}_{\text {cusp }}^{d}$ of $\mathbb{R} \mathcal{M}_{\text {cusp }}^{d} \cdot-$ The projection

$$
\pi_{\mathbb{R}}: \mathbb{R} \mathcal{M}_{\text {cusp }}^{d} \longrightarrow \mathbb{R} \mathcal{J}_{\omega} \times \mathbb{R}_{\tau} X^{\nu}
$$

is not proper in general. The reason for this is that there might exist some sequence $\left(\left[u_{n}, J_{S}^{n}, J^{n}, \underline{x}^{n}, z_{c}^{n}\right]\right)_{n \in \mathbb{N}}$ of $\mathbb{R} \mathcal{M}_{\text {cusp }}^{d}$ such that $\left(J^{n}, \underline{x}^{n}\right)$ converges to $\left(J^{\infty}, \underline{x}^{\infty}\right) \in \mathbb{R} \mathcal{J}_{\omega} \times \mathbb{R}_{\tau} X^{\nu}$, but the image $u_{n}(S)$ converges to some reducible $J^{\infty}$-holomorphic curve. From Gromov compactness theorem (see [5], Theorem 5.5.5), this is the only obstruction to the properness of $\pi_{\mathbb{R}}$. More precisely, this theorem describes how the sequence of maps $\left(u_{n}\right)_{n \in \mathbb{N}}$ does converge. There exist smooth disjoint loops $\alpha_{1}, \ldots, \alpha_{k}$ in $S$ and a map $u_{\infty}: S \rightarrow X$ which contracts the loops $\alpha_{1}, \ldots, \alpha_{k}$ and whose image is the reducible curve in the limit. Moreover, after may be changing the parameterization of $u_{n}$, this sequence converges to $u_{\infty}$ in $C^{0}$ norm on the whole $S$ and in norm $L^{k, p}$ on every compact subset of $S \backslash\left(\bigcup_{i=1}^{k} \alpha_{i}\right)$. In particular, we have the following alternative. Either the limit $z_{c}^{\infty}$ of $\left(z_{c}^{n}\right)_{n \in \mathbb{N}}$ does not belong to $\bigcup_{i=1}^{k} \alpha_{i}$, and then the curve in the limit has a cuspidal point at $u_{\infty}\left(z_{c}^{\infty}\right)$. Or the limit $z_{c}^{\infty}$ of $\left(z_{c}^{n}\right)_{n \in \mathbb{N}}$ does belong to $\bigcup_{i=1}^{k} \alpha_{i}$, say $\alpha_{1}$, and then the two irreducible components adjacent to $\alpha_{1}$ intersect each other with multiplicity at least 2 at $u_{\infty}\left(z_{c}^{\infty}\right)$. The latter can be obtained from adjunction formula for example, since the total sum of multiplicies of the singularities of the curve is controled by this formula.

The end of this paragraph is devoted to the proof that over a generic path

$$
\gamma: t \in[0,1] \longmapsto\left(J^{t}, \underline{x}^{t}\right) \in \mathbb{R}_{\mathcal{\omega}} \times \mathbb{R}_{\tau} X^{\nu},
$$

the only reducible curves which satisfy one of these two conditions have two irreducible components, both real, and only transversal double points as singularities with the exception of a unique real ordinary cusp or a unique real ordinary tacnode at some intersection point between the two irreducible components. Moreover, all these singularities are outside $\underline{x}^{t}$.

Let $m_{1} \in \mathbb{N}, d_{1} \in H_{2}(X ; \mathbb{Z})$ and $\underline{z}^{1}=\left(z_{1}^{1}, \ldots, z_{m_{1}}^{1}\right) \in S^{m_{1}}$ be an $m_{1}$-tuple of distinct points of $S$. Denote by

$$
\begin{aligned}
\mathbb{R} \mathcal{M}^{\left(d_{1}, m_{1}\right)}=\left\{\left(u^{1}, J_{S}^{1}, J^{1}, \underline{x}^{1}\right) \in L^{k, p}(S, X) \times \mathcal{J}_{S} \times \mathcal{J}_{\omega} \times X^{m_{1}}\right. \\
\left.\mid \mathrm{d} u^{1}+J^{1} \circ \mathrm{d} u^{1} \circ J_{S}^{1}=0, u\left(\underline{z}^{1}\right)=\underline{x}^{1}\right\} / \mathcal{D} \text { iff }{ }^{+}\left(S, \underline{z}^{1}\right) .
\end{aligned}
$$

Let $m_{2} \in \mathbb{N}, d_{2} \in H_{2}(X ; \mathbb{Z})$ and $\underline{z}^{2}=\left(z_{1}^{2}, \ldots, z_{m_{2}}^{2}\right) \in S^{m_{2}}$ be an $m_{2}$-tuple of distinct points of $S$. We denote by

$$
\mathbb{R} \mathcal{M}^{\left(d_{1}, m_{1}\right),\left(d_{2}, m_{2}\right)}=\left(\mathbb{R} \mathcal{M}^{\left(d_{1}, m_{1}\right)} \times \mathcal{J}_{\omega} \mathbb{R} \mathcal{M}^{\left(d_{2}, m_{2}\right)}\right) \backslash \text { Diag }
$$

where

$$
\begin{aligned}
& \operatorname{Diag}=\left\{\left(\left(u^{1}, J_{S}^{1}, J, \underline{x}^{1}\right),\left(u^{2}, J_{S}^{2}, J, \underline{x}^{2}\right)\right) \in \mathbb{R} \mathcal{M}^{\left(d_{1}, m_{1}\right)}\right. \times \mathcal{J}_{\omega} \mathbb{R} \mathcal{M}^{\left(d_{2}, m_{2}\right)} \\
&\left.\mid u_{1}(S)=u_{2}(S)\right\} .
\end{aligned}
$$

BULLETIN DE LA SOCIÉtÉ MATHÉMATIQUE DE FRANCE 
We recall the following proposition (see [11], Proposition 2.9 and Corollary 2.10).

Proposition 1.9. - The space $\mathbb{R} \mathcal{M}^{\left(d_{1}, m_{1}\right),\left(d_{2}, m_{2}\right)}$ is a separable Banach manifold of class $C^{\ell-k}$. Moreover, the projection

$$
\pi_{\mathbb{R}}^{\left(d_{1}, m_{1}\right),\left(d_{2}, m_{2}\right)}: \mathbb{R} \mathcal{M}^{\left(d_{1}, m_{1}\right),\left(d_{2}, m_{2}\right)} \longrightarrow \mathbb{R} \mathcal{J}_{\omega} \times \mathbb{R}_{\tau_{1}} X^{m_{1}} \times \mathbb{R}_{\tau_{2}} X^{m_{2}}
$$

is Fredholm of index $\nu-m_{1}-m_{2}$ where $d=d_{1}+d_{2}$. Finally, the cokernel of $\pi_{\mathbb{R}}^{\left(d_{1}, m_{1}\right),\left(d_{2}, m_{2}\right)}$ at $\left(\left(u^{1}, J_{S}^{1}, J, \underline{x}^{1}\right),\left(u^{2}, J_{S}^{2}, J, \underline{x}^{2}\right)\right)$ is isomorphic to

$$
H_{D}^{1}\left(S, N_{u_{1},-\underline{z}^{1}}\right)_{+1} \oplus H_{D}^{1}\left(S, N_{u_{2},-\underline{z}^{2}}\right)_{+1} \text {. }
$$

REMARK 1.10. - Over a generic path $\gamma: t \in[0,1] \mapsto\left(J^{t}, \underline{x}^{t}\right) \in \mathbb{R} \mathcal{J}_{\omega} \times \mathbb{R}_{\tau} X^{\nu}$, no $J^{t}$-holomorphic curve which realize $d$ and pass through $\underline{x}^{t}$ can be multiple or come from the diagonal Diag. Indeed, the condition for a $J$-holomorphic curve which realize the homology class $\frac{1}{2} d$ to pass through $\nu$ distinct points is of codimension $\frac{1}{2} c_{1}(X) d-1$, that is of codimension greater than 1 as soon as $c_{1}(X) d>4$. Moreover, a generic immersed rational $J$-holomorphic curve which realize the homology class $\frac{1}{2} d$ has $\frac{1}{2}\left(\left(\frac{d}{2}\right)^{2}-\frac{1}{2} c_{1}(X) d+2\right)$ transversal double points. Each of these double points is responsible for four double points of the doubled curve. The number of double points of the doubled curve would then be at least $2\left(\left(\frac{d}{2}\right)^{2}-\frac{1}{2} c_{1}(X) d+2\right)-1$, which is impossible as soon as $c_{1}(X) d<4$.

Proposition 1.11. - The subspace of $\mathbb{R} \mathcal{M}^{\left(d_{1}, m_{1}\right),\left(d_{2}, m_{2}\right)}$ made of couples $\left(\left(u^{1}, J_{S}^{1}, J, \underline{x}^{1}\right),\left(u^{2}, J_{S}^{2}, J, \underline{x}^{2}\right)\right)$ for which $u^{1}$ or $u^{2}$ has a unique cuspidal point which is ordinary, or for which $u^{1}(S)$ and $u^{2}(S)$ have a unique point of contact which is of order 2 , all the singularities of $u^{1}(S) \cup u^{2}(S)$ being outside $\underline{x}^{1} \cup \underline{x}^{2}$ is a stratum of codimension 1. The subspace of curves having degenerated cuspidal points or higher order cuspidal points, or points of contact of higher order is a stratum of codimension at least 2.

We denote by $\mathbb{R} \mathcal{M}^{\left(d_{1}, m_{1}\right),\left(d_{2}, m_{2}\right)} \operatorname{cusp}\left(\operatorname{resp} . \mathbb{R} \mathcal{M}_{\text {tac }}^{\left(d_{1}, m_{1}\right),\left(d_{2}, m_{2}\right)}\right.$ ) the codimension 1 stratum of $\mathbb{R} \mathcal{M}^{\left(d_{1}, m_{1}\right),\left(d_{2}, m_{2}\right)}$ given by Proposition 1.11 made of curves having a real ordinary cusp (resp. an ordinary point of contact between $u^{1}(S)$ and $\left.u^{2}(S)\right)$.

Corollary 1.12. - Let $\gamma: t \in[0,1] \mapsto\left(J^{t}, \underline{x}^{t}\right) \in \mathbb{R} \mathcal{J}_{\omega} \times \mathbb{R}_{\tau} X^{\nu}$ be a generic path. Assume that a sequence of elements of $\mathbb{R} \mathcal{M}_{\text {cusp }}^{d}$ over $\gamma$ converges to some reducible curve. Then this reducible $J^{t}$-holomorphic curve is given by an element

$$
\left(\left[u^{1}, J_{S}^{1}, J, \underline{x}^{1}\right],\left[u^{2}, J_{S}^{2}, J, \underline{x}^{2}\right]\right) \in \mathbb{R} \mathcal{M}^{\left(d_{1}, m_{1}\right),\left(d_{2}, m_{2}\right)} \operatorname{cusp} \cup \mathbb{R} \mathcal{M}_{\mathrm{tac}}^{\left(d_{1}, m_{1}\right),\left(d_{2}, m_{2}\right)}
$$
such that $d_{1}+d_{2}=d, m_{1}+m_{2}=\nu$ and $\left.t \in\right] 0,1\left[\right.$. Moreover, either $m_{1}=$ $c_{1}(X) d_{1}-1$, or $m_{1}=c_{1}(X) d_{1}-2$ and then the cuspidal point, if it exists, belongs to $u^{1}(S)$. 
Proof. - From Proposition 1.11 follows that the curve in the limit must belong to $\mathbb{R} \mathcal{M}_{\text {cusp }}^{\left(d_{1}, m_{1}\right),\left(d_{2}, m_{2}\right)} \cup \mathbb{R} \mathcal{M}_{\text {tac }}^{\left(d_{1}, m_{1}\right),\left(d_{2}, m_{2}\right)}$ as soon as $\gamma$ is generic enough. Now, from Proposition 1.9, the cokernel of $\mathrm{d} \pi_{\mathbb{R}}^{\left(d_{1}, m_{1}\right),\left(d_{2}, m_{2}\right)} \mathrm{I}\left(\left[u^{1}, J_{S}^{1}, J, \underline{x}^{1}\right],\left[u^{2}, J_{S}^{2}, J, \underline{x}^{2}\right]\right)$ is isomorphic to $H_{D}^{1}\left(S, N_{u_{1},-\underline{z}^{1}}\right)_{+1} \oplus H_{D}^{1}\left(S, N_{u_{2},-\underline{z}^{2}}\right)_{+1}$. Since this cokernel is of dimension less than 2 , we have $m_{i} \leq c_{1}(X) d_{i}$ for $1 \leq i \leq 2$. Moreover, in case $m_{2}=c_{1}(X) d_{2}$, we have $\operatorname{dim} H_{D}^{1}\left(S, N_{u_{2},-\underline{z}^{2}}\right)_{+1} \geq 1$ with equality if and only if the map $u^{2}$ is an immersion (see [2]). Finally, the relation $m_{1}+m_{2}=\nu$ forces each irreducible component in the limit to be simply covered, unless $c_{1}(X) d_{i} \leq 1$ for some $i \in\{1,2\}$. Now, when $c_{1}(X) d_{i} \leq 1$ for some $i \in\{1,2\}$, it suffices to count the number of double points of these rational curves as in Remark 1.10 to see that these irreducible components cannot be multiply covered.

REMARK 1.13. - If we would not have excluded the case $r=(0, \ldots, 0)$, then a sequence of real rational cuspidal $J$-holomorphic curves could converge to a reducible curve having two irreducible components which are complex conjugated and transversal to each other except at one point which is of order two, that is an ordinary tacnode.

\section{Study of degenerated order two cuspidal points}

2.1. Local study of degenerated order two cuspidal points. - Let $B^{4}$ be the unit ball of $\mathbb{C}^{2}$ and $c_{X}$ be the restriction of the complex conjugation to $B^{4}$. Denote by $\mathbb{R} B^{4}$ the fixed point set of $c_{X}$; it is the unit ball of $\mathbb{R}^{2} \subset \mathbb{C}^{2}$. Denote by $\mathbb{R} \mathcal{J}_{\omega_{\mathrm{st}}}$ the space of almost complex structures $J$ of $B^{4}$ which are tamed by the standard symplectic form $\omega_{\text {st }}$ and for which $c_{X}$ is $J$ antiholomorphic. Let $\bar{B}^{2}$ be the closed unit ball of $\mathbb{C}$ and conj the restriction of the complex conjugation to $\bar{B}^{2}$. Its fixed point set is $]-1,1\left[\subset \bar{B}^{2}\right.$.

Finally, denote by $J_{\text {st }}$ the restriction of the complex structure of $\mathbb{C}^{2}$ to $B^{4}$, so that $J_{\text {st }} \in \mathbb{R} \mathcal{J}_{\omega_{\mathrm{st}}}$. Let $\eta>0$ and

$$
\begin{array}{r}
\mathbb{R}_{\text {cusp }}^{\prime}(\eta)=\left\{\left(u, J, z_{c}\right) \in L^{k, p}\left(\bar{B}^{2}, B^{4}\right) \times \mathbb{R} \mathcal{J}_{\omega_{\mathrm{st}}} \times\right]-1,1[ \\
\mid\left\|J-J_{\mathrm{st}}\right\|_{C^{1}}<\eta, \mathrm{d} u+J \circ \mathrm{d} u \circ i=0 \\
c_{X} \circ u=u \circ \operatorname{conj}, \mathrm{d}_{z_{c}} u=0 \text { but }\left.\nabla \mathrm{d} u\right|_{z_{c}} \neq 0 \\
\text { and } \left.u\left(\bar{B}^{2}\right) \text { has smooth boundary }\right\} .
\end{array}
$$

In particular, $u$ is not multiple. Note that $\mathbb{R} \mathcal{P}_{\text {cusp }}^{\prime}(\eta)$ is not connected. Indeed, two disks which do not have the same number of double points cannot be in the same connected component. We are in fact interested here in a connected component for which general elements are disks with one ordinary cusp at $z_{c}$ and one transversal double point. 
LEMMA 2.1. - As soon as $\eta$ is small enough, $\mathbb{R}_{\text {cusp }}^{\prime}(\eta)$ is a separable Banach manifold of class $C^{\ell-k}$ whose tangent space at $\left(u, J, z_{c}\right)$ is

$$
\begin{aligned}
& T_{\left(u, J, z_{c}\right)} \mathbb{R} \mathcal{P}_{\text {cusp }}^{\prime}(\eta)=\left\{\left(v, \dot{J}, \dot{z}_{c}\right) \in L^{k, p}\left(\bar{B}^{2}, \mathbb{C}^{2}\right) \times T_{J} \mathbb{R} \mathcal{J}_{\omega_{\text {st }}} \times \mathbb{R}\right. \\
& \mid D v+\dot{J} \circ \mathrm{d} u \circ i=0, v=\mathrm{d} c_{X} \circ v \circ \text { conj }, \\
&\left.\nabla v_{z_{z_{c}}}+\nabla_{\dot{z}_{c}} \mathrm{~d} u=0\right\} .
\end{aligned}
$$

Let $\left(u, J, z_{c}\right) \in \mathbb{R} \mathcal{P}_{\text {cusp }}^{\prime}(\eta)$, then the order 3 jet of $u$ at the point $z_{c}$ is a complex polynomial which can be written

$$
u\left(z_{c}\right)+j_{2}\left(z_{c}\right)\left(z-z_{c}\right)^{2}+j_{3}\left(z_{c}\right)\left(z-z_{c}\right)^{3}
$$

with $0 \neq j_{2}\left(z_{c}\right)=\frac{1}{2} \frac{\partial^{2} u}{\partial z^{2}} \in \mathbb{R}^{2}$ and $j_{3}\left(z_{c}\right)=\frac{1}{6} \frac{\partial^{3} u}{\partial z^{3}} \in \mathbb{R}^{2}$ (see [8], Proposition 3). The cuspidal point $z_{c}$ is degenerated when $j_{3}\left(z_{c}\right)$ is colinear to $j_{2}\left(z_{c}\right)$. Let

$$
F_{\text {deg }}:\left(u, J, z_{c}\right) \in \mathbb{R} \mathcal{P}_{\text {cusp }}^{\prime}(\eta) \longmapsto \operatorname{det}\left(j_{2}\left(z_{c}\right), j_{3}\left(z_{c}\right)\right) \in \mathbb{R},
$$

and $\mathbb{R} \mathcal{P}_{\text {dcusp }}^{\prime}(\eta)=F_{\text {deg }}^{-1}(0)$ be the locus of curves $\left(u, J, z_{c}\right)$ having a degenerated cuspidal point at $z_{c}$.

Lemma 2.2. - As soon as $\eta$ is small enough, $\mathbb{R} \mathcal{P}_{\text {dcusp }}^{\prime}(\eta)$ is a separable Banach submanifold of class $C^{\ell-k}$ of $\mathbb{R} \mathcal{P}_{\text {cusp }}^{\prime}(\eta)$ whose tangent space at $\left(u, J, z_{c}\right)$ is

$$
\begin{aligned}
T_{\left(u, J, z_{c}\right)} \mathbb{R} \mathcal{P}_{\text {dcusp }}^{\prime}(\eta)=\left\{\left(v, \dot{J}, \dot{z}_{c}\right)\right. & \in L^{k, p}\left(\bar{B}^{2}, \mathbb{C}^{2}\right) \times T_{J} \mathbb{R} \mathcal{J}_{\omega_{\mathrm{st}}} \times \mathbb{R} \\
\mid \operatorname{det}( & \left.d_{\left(v, \dot{J}, \dot{z}_{c}\right)} j_{2}\left(z_{c}\right), j_{3}\left(z_{c}\right)\right) \\
& \left.+\operatorname{det}\left(j_{2}\left(z_{c}\right), d_{\left(v, j, \dot{z}_{c}\right)} j_{3}\left(z_{c}\right)\right)=0\right\} .
\end{aligned}
$$

Proof. - The function $F_{\mathrm{deg}}$ is of class $C^{\ell-k}$ as soon as $k \geq 3$ since $u$ is of class $C^{\ell}$. It suffices to prove that $\mathrm{d}_{\left(u, J, z_{c}\right)} F_{\text {deg }}$ is surjective at each point $\left(u, J, z_{c}\right)$ of $\mathbb{R} \mathcal{P}_{\text {dcusp }}^{\prime}(\eta)$. Let $\left(u_{0}, J_{0}, z_{c}\right)$ be such a point. From Lemma 2.5 of [11], there exists a smooth family of $J_{0}$-holomorphic maps $\left(\tilde{u}_{\lambda}\right)_{\lambda \in]-\epsilon, \epsilon[}$ defined in a neighbourhood $U$ of $z_{c}$ by

$$
\tilde{u}_{\lambda}(z)=u_{0}(z)+\left(z-z_{c}\right)^{3}\left(\lambda w+w_{\lambda}(z)\right),
$$

where $w$ can be any vector in $\mathbb{R}^{2}$ and $w_{\lambda} \in L^{k, p}\left(\bar{B}^{2}, \mathbb{C}^{2}\right)$ is real and satisfies $w_{0}=0, \frac{\mathrm{d}}{\mathrm{d} \lambda} w_{\lambda \mid \lambda=0}=0$. This family can be extended to a smooth family $\left(u_{\lambda}, J_{\lambda}, z_{c}\right)_{\lambda \in]-\epsilon, \epsilon[} \in \mathbb{R} \mathcal{P}_{\text {cusp }}^{\prime}(\eta)$ such that $J_{\lambda}=J_{0}$ if $\lambda=0$ and $J_{\lambda}$ differs from $J_{0}$ only in a neighbourhood of $u_{0}(\partial U)$. Indeed, it suffices to glue the map $\tilde{u}_{\lambda \mid U}$ to the map $u_{0 \mid} \bar{B}^{2} \backslash U$ with the help of an annulus embedded in a neighbourhood of $u_{0}(\partial U)$. The obtained map can be made $J_{\lambda}$-holomorphic for some $J_{\lambda}$ which equals $J_{0}$ outside a neighbourhood of $u_{0}(\partial U)$. We have then $\frac{\mathrm{d}}{\mathrm{d} \lambda} j_{2}\left(u_{\lambda}, z_{c}\right)=0$ and $\frac{\mathrm{d}}{\mathrm{d} \lambda} j_{3}\left(u_{\lambda}, z_{c}\right)=w$. Hence, $\mathrm{d}_{\left(\dot{\tilde{u}}_{\lambda}, \dot{J}_{\lambda}, z_{c}\right)} F_{\operatorname{deg}}=\operatorname{det}\left(j_{2}\left(z_{c}\right), w\right)$ does not vanish as soon as $w$ is not chosen colinear to $j_{2}\left(z_{c}\right)$.

TOME $134-2006-\mathrm{N}^{\mathrm{O}} 2$ 


\section{REMARK 2.3}

1) The group of real biholomorphisms of $\left(\bar{B}^{2}, i\right)$ acts on $\mathbb{R} \mathcal{P}_{\text {cusp }}^{\prime}(\eta)$ by

$$
\phi \cdot\left(u, J, z_{c}\right)=\left(u \circ \phi^{-1}, J, \phi\left(z_{c}\right)\right) .
$$

Since it is transitive on ] $-1,1\left[\right.$, we can always assume that $z_{c}=0$.

$2)$ Let $(u, J, 0) \in \mathbb{R} \mathcal{P}_{\text {dcusp }}^{\prime}(\eta)$ and $a \in \mathbb{R}$ be such that $j_{3}(u, 0)=a j_{2}(u, 0)$. Let $z=w-\frac{1}{2} a w^{2}$, the order 3 jet of $u$ writes

$$
\begin{aligned}
u(z) & =j_{2}(u, 0)\left(w-\frac{a}{2} w^{2}\right)^{2}+j_{3}(u, 0)\left(w-\frac{a}{2} w^{2}\right)^{3}+o\left(|w|^{3}\right) \\
& =j_{2}(u, 0) w^{2}+o\left(|w|^{3}\right) .
\end{aligned}
$$

Lemma 2.4. - Let $(u, J, 0) \in \mathbb{R} \mathcal{P}_{\text {dcusp }}^{\prime}(\eta)$ and $a \in \mathbb{R}$ be such that $j_{3}(u, 0)=$ $a j_{2}(u, 0)$. Let $z=w-\frac{1}{2} a w^{2}$, the order 5 germ of $u$ writes

$$
u(z)=j_{2} w^{2}+j_{4}^{w} w^{4}+j_{4}^{|w|}|w|^{4}+j_{4}^{\bar{w}} \bar{w}^{4}+j_{5} w^{5}+o\left(|w|^{5}\right),
$$

where $j_{2}, j_{4}^{w}, j_{4}^{|w|}, j_{4}^{\bar{w}}, j_{5} \in \mathbb{R}^{2}$.

Proof. - This is deduced after expanding the relation $0=J_{\mid u} \circ \mathrm{d} u-\mathrm{d} u \circ i$.

A degenerated cuspidal curve $\left(u, J, z_{c}\right) \in \mathbb{R}^{\prime}{ }_{\text {dcusp }}(\eta)$ as in Lemma 2.4 is said to be generic if the vectors $j_{2}$ and $j_{5}$ are linearly independant.

Proposition 2.5. - Let $(u, J, 0) \in \mathbb{R}_{\text {dcusp }}^{\prime}(\eta)$ be a generic cuspidal curve and $v(z)=d_{z} u\left(\frac{1}{z}\right)$. Then $(v, 0,0) \in T_{(u, J, 0)} \mathbb{R}_{\text {cusp }}^{\prime}(\eta) \backslash T \mathbb{R} \mathcal{P}_{\text {dcusp }}^{\prime}(\eta)$. Moreover, if $\left(u_{\lambda}, J_{\lambda}, z_{c}^{\lambda}\right)_{\lambda \in]-\epsilon, \epsilon[}$ is a path of $\mathbb{R} \mathcal{P}_{\text {cusp }}^{\prime}(\eta)$ transversal to $\mathbb{R} \mathcal{P}_{\text {dcusp }}^{\prime}$ at $\lambda=0$, then for $\lambda<0$ (resp. $\lambda>0$ ) close enough to $0, u_{\lambda}$ has an isolated (resp. non isolated) real double point in a neighbourhood of the cusp $u_{\lambda}\left(z_{c}^{\lambda}\right)$, or vice versa.

Proof. - With the notations of Lemma 2.4, we make the local change of coordinates $z=\phi(w)$ with $\phi(w)=w-\frac{1}{2} a w^{2}$. The order five germ of $u$ writes

$$
u \circ \phi(w)=j_{2} w^{2}+j_{4}^{w} w^{4}+j_{4}^{|w|}|w|^{4}+j_{4}^{\bar{w}} \bar{w}^{4}+j_{5} w^{5}+o\left(|w|^{5}\right),
$$

where $j_{2}, j_{4}^{w}, j_{4}^{|w|}, j_{4}^{\bar{w}}, j_{5} \in \mathbb{R}^{2}$. Equip $\mathbb{C}^{2}$ with the complex structure $J(0)$ and the frame $\left(u_{0}(0), j_{2}, j_{5}\right)$. After composition with the local diffeomorphism of $\mathbb{C}^{2}$ tangent to the identity given by

$$
\Phi\left(z_{1}, z_{2}\right)=\left(z_{1}, z_{2}\right)-j_{4}^{w} z_{1}^{2}-j_{4}^{|w|}\left|z_{1}\right|^{2}-j_{4}^{\bar{w}} \bar{z}_{1}^{2},
$$

we can assume that the jet of $u$ writes $j_{2} w^{2}+j_{5} w^{5}+o\left(|w|^{5}\right)$. It suffices to prove that

$$
\tilde{v}=\mathrm{d}_{w}(\Phi \circ u \circ \phi)\left(\frac{1}{w}\right) \in T_{(u \circ \phi, J, 0)} \mathbb{R} \mathcal{P}_{\text {cusp }}^{\prime}(\eta) \backslash T \mathbb{R} \mathcal{P}_{\text {dcusp }}^{\prime}(\eta) .
$$

BULLETIN DE LA SOCiÉTÉ MATHÉMATIQUe DE FRANCE 
Indeed, $\mathrm{d}_{w}(u \circ \phi)\left(\frac{1}{w}\right)=\mathrm{d}_{z} u\left(\frac{1}{z}+b(z)\right)$ for some holomorphic $b$, and $\mathrm{d}_{z} u(b(z)) \in$ $T \mathbb{R} \mathcal{P}_{\text {dcusp }}^{\prime}(\eta)$. Moreover, the composition by $\Phi$ does not affect the transversality condition. Now $\tilde{v}(w)=2 j_{2}+5 j_{5} w^{3}+o\left(|w|^{3}\right)$. From Lemma 2.1, $(\tilde{v}, 0,0) \in$ $T_{(u \circ \phi, J, 0)} \mathbb{R} \mathcal{P}_{\text {cusp }}^{\prime}(\eta)$ and from Lemma $2.2,(\tilde{v}, 0,0) \notin T_{(u \circ \phi, J, 0)} \mathbb{R} \mathcal{P}_{\text {dcusp }}^{\prime}(\eta)$, since $\mathrm{d} j_{3}(\tilde{v}, 0,0)=5 j_{5}$ and $\operatorname{det}\left(j_{2}, 5 j_{5}\right) \neq 0$. The first part of the proposition is proved.

Now, without loss of generality, we can assume that $\frac{\mathrm{d}}{\mathrm{d} \lambda}\left(u_{\lambda}, J_{\lambda}, z_{c}^{\lambda}\right)=(v, 0,0)$ and $z_{c}^{\lambda} \equiv 0$. From what precedes, the expansion of $u_{\lambda}\left(w-\frac{1}{2} a w^{2}\right)$ writes

$u_{\lambda}\left(w-\frac{1}{2} a w^{2}\right) f(\lambda)+\left(w^{2}, w^{5}\right)+O\left(|w|^{6}\right)+\lambda\left(0,5 w^{3}\right)+O\left(\lambda|w|^{4}\right)+O\left(\lambda^{2}|w|^{2}\right)$.

The function $f(\lambda)+\left(w^{2}, 5 \lambda w^{3}+w^{5}\right)$ has an isolated real double point at the parameters $w= \pm i \sqrt{5 \lambda}$ when $\lambda>0$. Let us prove that when $\lambda>0$ is close enough to 0 , the function $u_{\lambda}\left(w-\frac{1}{a} 2 w^{2}\right)$ also has an isolated real double point at parameters close to $\pm i \sqrt{5 \lambda}$. Set $w=i \sqrt{5 \lambda}+\tilde{w}$, we have

$$
\begin{array}{r}
u_{\lambda} \circ \phi(i \sqrt{5 \lambda}+\tilde{w})=f(\lambda)+(-5 \lambda, 0)+\left(2 i \sqrt{5 \lambda} \tilde{w}+\tilde{w}^{2}+\lambda^{2} O(|\tilde{w}|+|\lambda|),\right. \\
\left.50 \lambda^{2} \tilde{w}+|\lambda|^{\frac{3}{2}} \tilde{w}^{2} O(|\tilde{w}|+|\lambda|)+\lambda^{3} O(|\tilde{w}|+|\lambda|)\right),
\end{array}
$$

as soon as $|\tilde{w}| \ll \sqrt{\lambda}$. Set $\tilde{w}=|\lambda|^{\frac{3}{4}}(\cos (\theta)+i \sin (\theta))$, we get

$$
\begin{array}{r}
\operatorname{Im}\left(u_{\lambda} \circ \phi(i \sqrt{5 \lambda}+\tilde{w})\right)=\left(2 \sqrt{5}|\lambda|^{\frac{5}{4}} \cos (\theta)+|\lambda|^{\frac{3}{2}} O(|\lambda|),\right. \\
\left.50|\lambda|^{\frac{11}{4}} \sin (\theta)+|\lambda|^{3} O(|\lambda|)\right) .
\end{array}
$$

The linking number between the origin of $\mathbb{R}^{2}$ and the ellipse parameterized by $\theta \mapsto\left(2 \sqrt{5}|\lambda|^{\frac{5}{4}} \cos (\theta), 50|\lambda|^{\frac{11}{4}} \sin (\theta)\right)$ is equal to 1 . The same result holds for the linking number between the origin of $\mathbb{R}^{2}$ and the curve parameterized by $\theta \mapsto \operatorname{Im}\left(u_{\lambda} \circ \phi\left(i \sqrt{5 \lambda}+|\lambda|^{\frac{3}{4}}(\cos (\theta)+i \sin (\theta))\right)\right)$ as soon as $\lambda>0$ is close enough to 0 . Hence, $\operatorname{Im}\left(u_{\lambda} \circ \phi\right)$ vanishes once in the disk centered at $i \sqrt{5 \lambda}$ and whose radius is $|\lambda|^{\frac{3}{4}}$. It follows that $u_{\lambda}$ has an isolated real double point close to its cuspidal point $u_{\lambda}(0)$ as soon as $\lambda>0$ is close enough to 0 .

In the same way, the function $f(\lambda)+\left(w^{2}, 5 \lambda w^{3}+w^{5}\right)$ has a non isolated real double point at the parameters $w= \pm \sqrt{-5 \lambda}$ when $\lambda<0$. Let us prove that when $\lambda<0$ is close enough to 0 , the function $u_{\lambda}\left(w-\frac{a}{2} w^{2}\right)$ also has a non isolated real double point at parameters close to $\pm \sqrt{-5 \lambda}$. Set $w=\eta \sqrt{-5 \lambda}+\tilde{w}$, where $\eta= \pm 1$, we have

$$
\begin{aligned}
u_{\lambda} \circ \phi(\eta \sqrt{-5 \lambda}+\tilde{w})=f(\lambda) & +(-5 \lambda, 0) \\
+ & \left(2 \eta \sqrt{-5 \lambda} \tilde{w}+\tilde{w}^{2}+|\lambda|^{2} O(|\tilde{w}|+|\lambda|),\right. \\
& \left.50 \lambda^{2} \tilde{w}+|\lambda|^{\frac{3}{2}} \tilde{w}^{2} O(|\tilde{w}|+|\lambda|)+\lambda^{3} O(|\tilde{w}|+|\lambda|)\right),
\end{aligned}
$$

as soon as $|\tilde{w}| \ll \sqrt{|\lambda|}$. When $\tilde{w} \in\left[-|\lambda|^{\frac{3}{4}},|\lambda|^{\frac{3}{4}}\right]$, the segment $\left(2 \eta \sqrt{-5 \lambda} \tilde{w}, 50 \lambda^{2} \tilde{w}\right)$ joins the two points $\left(-2 \sqrt{5} \eta|\lambda|^{\frac{5}{4}},-50|\lambda|^{\frac{11}{4}}\right)$ and $\left(2 \sqrt{5} \eta|\lambda|^{\frac{5}{4}}, 50|\lambda|^{\frac{11}{4}}\right)$. When

TOME $134-2006-\mathrm{N}^{\mathrm{O}} 2$ 
$\eta= \pm 1$, these two segments intersect transversely at the origin. The same is true for the segments $u_{\lambda} \circ \phi\left(\eta \sqrt{-5 \lambda}+\left[-|\lambda|^{\frac{3}{4}},|\lambda|^{\frac{3}{4}}\right]\right)$ as soon as $\lambda<0$ is close enough to 0 , hence the result.

2.2. Global study of degenerated order two cuspidal points. - Denote by $\mathbb{R} \mathcal{M}_{\text {dcusp }}^{d}$ the subset of elements $\left[u, J_{S}, J, \underline{x}, z_{c}\right]$ of $\mathbb{R} \mathcal{M}_{\text {cusp }}^{d}$ for which $u$ has a degenerated cuspidal point at $z_{c}$.

Proposition 2.6. - The space $\mathbb{R} \mathcal{M}_{\mathrm{dcusp}}^{d}$ is a codimension one submanifold of $\mathbb{R} \mathcal{M}_{\text {cusp }}^{d}$ of class $C^{\ell-k}$. Moreover, the subspace of $\mathbb{R} \mathcal{M}_{\mathrm{dcusp}}^{d}$ made of curves $\left[u, J_{S}, J, \underline{x}, z_{c}\right]$ which have a non generic degenerated cuspidal point at $z_{c}$ is a substratum of codimension two and class $C^{\ell-k}$ of $\mathbb{R} \mathcal{M}_{\text {cusp }}^{d}$. of $[1]$.)

(This is a particular case of Theorem 3.4.5 of [6], see also Proposition 7

Let $\gamma:[0,1] \rightarrow \mathbb{R} \mathcal{J}_{\omega} \times \mathbb{R}_{\tau} X^{c_{1}(X) d-2}$ be a path transversal to $\pi_{\mathbb{R}}$. From Proposition 2.6, if it is chosen generic enough, it avoids the image under $\pi_{\mathbb{R}}$ of curves having a non generic degenerated cuspidal point. Denote by

$$
\mathbb{R} \mathcal{M}_{\gamma}=\mathbb{R} \mathcal{M}_{\text {cusp }}^{d} \times_{\gamma}[0,1]
$$

and by $\pi_{\gamma}: \mathbb{R} \mathcal{M}_{\gamma} \rightarrow[0,1]$ the associated projection.

Proposition 2.7. - Let $C_{t_{0}}=\left[u, J_{S}, J^{t_{0}}, \underline{x}^{t_{0}}, z_{c}\right] \in \mathbb{R} \mathcal{M}_{\gamma}$ be a curve having a degenerated cuspidal point at $z_{c}$. Then, there exists a neighbourhood $W$ of $\left[u, J_{S}, J^{t_{0}}, \underline{x}^{t_{0}}, z_{c}\right]$ in $\mathbb{R} \mathcal{M}_{\gamma}$ and $\epsilon>0$ such that for every $\left.t \in\right] t_{0}-\epsilon, t_{0}[$, $\pi_{\gamma}^{-1}(t) \cap W$ is made of two curves $C_{t}^{+}, C_{t}^{-}$such that $m\left(C_{t}^{+}\right)=m\left(C_{t}^{-}\right)+1$ and for every $t \in] t_{0}, t_{0}+\epsilon\left[, \pi_{\gamma}^{-1}(t) \cap W=\varnothing\right.$, or vice versa.

Proof. - From Proposition 2.6, the degenerated cuspidal point of $C_{t_{0}}$ is generic. From Theorem 1.8, $C_{t_{0}}$ is a non-degenerated critical point of $\pi_{\gamma}$. Thus, as soon as $\epsilon$ and $W$ are small enough, for every $t \in] t_{0}-\epsilon, t_{0}[$, $\pi_{\gamma}^{-1}(t) \cap W=\left\{C_{t}^{ \pm}\right\}$and for every $\left.t \in\right] t_{0}, t_{0}+\epsilon\left[, \pi_{\gamma}^{-1}(t) \cap W=\varnothing\right.$, or vice versa. The only thing to prove is that $m\left(C_{t}^{+}\right)=m\left(C_{t}^{-}\right)+1$. The double points of $C_{t}^{ \pm}$are close to the ones of $C_{t_{0}}$ with the exception of one which is close to the cusp of $C_{t_{0}}$. We have to prove that the nature of the latter is not the same for $C_{t}^{+}$and $C_{t}^{-}$. Note that $\left.\frac{\mathrm{d}}{\mathrm{d} t}\right|_{t=t_{0}} C_{t}$ is the generator of $\operatorname{ker} \mathrm{d} \pi_{\mathbb{R}}=H^{0}\left(S, \mathcal{N}_{u,-\underline{z}}^{\text {cusp }}\right)_{+1} \cong H^{0}\left(S, \mathbb{C}_{z_{c},-\underline{z}}\right)_{+1}$, see Proposition 1.2. Choose a neighbourhood $V$ of $u\left(z_{c}\right)$ invariant under $c_{X}$, diffeomorphic to the 4-ball $B^{4}$ and small enough in order that $\left\|J^{t}-J^{t_{0}}\left(u\left(z_{c}\right)\right)\right\|_{C^{1}}<\eta$. We deduce a restriction map rest $: W \subset \mathbb{R} \mathcal{M}_{\gamma} \rightarrow \mathbb{R} \mathcal{P}^{\prime} \operatorname{cusp}(\eta)$ such that $\operatorname{rest}\left(C_{t_{0}}\right) \in \mathbb{R} \mathcal{P}_{\text {dcusp }}^{\prime}(\eta)$. Now $\mathrm{d}_{C_{t_{0}}} \operatorname{rest}\left(\left.\frac{\mathrm{d}}{\mathrm{d} t}\right|_{t=t_{0}} C_{t}\right)$ is exactly the vector $(v, 0,0)$ given by Proposition 2.5 . The result thus follows from Proposition 2.5. 


\section{Study of the compactification $\mathbb{R} \overline{\mathcal{M}}_{\text {cusp }}^{d}$}

3.1. Neighbourhood of $\mathbb{R} \mathcal{M}_{\mathrm{tac}}^{\left(d_{1}, m_{1}\right),\left(d_{2}, m_{2}\right)}$ in $\mathbb{R} \overline{\mathcal{M}}_{\text {cusp }}^{d}$ when $m_{i}=$ $c_{1}(X) d_{i}-1$

Lemma 3.1. - Let $d_{1}, d_{2} \in H_{2}(X ; \mathbb{Z})$ be such that

$$
d_{1}+d_{2}=d \quad \text { and } \quad m_{i}=c_{1}(X) d_{i}-1, i \in\{1,2\} .
$$

Then, $\mathbb{R} \mathcal{M}_{\mathrm{tac}}^{\left(d_{1}, m_{1}\right),\left(d_{2}, m_{2}\right)}$ has a canonical coorientation in $\mathbb{R} \mathcal{M}^{\left(d_{1}, m_{1}\right),\left(d_{2}, m_{2}\right)}$. $A$ path $\left(C^{t}\right)_{t \in]-\epsilon, \epsilon[}$ in $\mathbb{R} \mathcal{M}^{\left(d_{1}, m_{1}\right),\left(d_{2}, m_{2}\right)}$ transversal to $\mathbb{R} \mathcal{M}_{\mathrm{tac}}^{\left(d_{1}, m_{1}\right),\left(d_{2}, m_{2}\right)}$ at $t=0$ is positive on this coorientation if for every $t \in]-\epsilon, 0[$ (resp. $t \in] 0, \epsilon[)$, $C^{t}$ has two real (resp. complex conjugated) double points in a neighbourhood of the tacnode of $C^{0}$.

(See $\S 1.5$ for the definition of the space $\mathbb{R} \mathcal{M}_{\text {tac }}^{\left(d_{1}, m_{1}\right),\left(d_{2}, m_{2}\right)}$.)

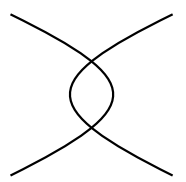

$C^{t}, t<0$

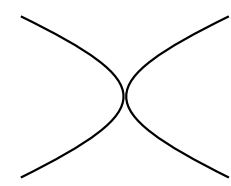

$C^{0}$

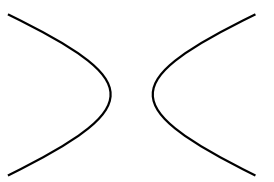

$C^{t}, t>0$

Figure 1

Remember that under the conditions of Lemma 3.1, the projection

$$
\pi_{\mathbb{R}}^{\left(d_{1}, m_{1}\right),\left(d_{2}, m_{2}\right)}: \mathbb{R} \mathcal{M}^{\left(d_{1}, m_{1}\right),\left(d_{2}, m_{2}\right)} \longrightarrow \mathbb{R} \mathcal{J}_{\omega} \times \mathbb{R}_{\tau_{1}} X^{m_{1}} \times \mathbb{R}_{\tau_{2}} X^{m_{2}}
$$

is Fredholm with vanishing index. Moreover, $\mathbb{R} \mathcal{M}_{\text {tac }}^{\left(d_{1}, m_{1}\right),\left(d_{2}, m_{2}\right)}$ is made of regular points of this projection, from Proposition 1.9. Let

$$
\gamma: t \in[0,1] \longmapsto\left(J^{t}, \underline{x}^{t}\right) \in \mathbb{R} \mathcal{J}_{\omega} \times \mathbb{R}_{\tau_{1}} X^{m_{1}} \times \mathbb{R}_{\tau_{2}} X^{m_{2}}
$$

be a path transversal to $\pi_{\mathbb{R}}^{\left(d_{1}, m_{1}\right),\left(d_{2}, m_{2}\right)}$, so that the fibre product $\mathbb{R} \mathcal{M}_{\gamma}^{\text {red }}=$ $\mathbb{R} \mathcal{M}^{\left(d_{1}, m_{1}\right),\left(d_{2}, m_{2}\right)} \times_{\pi_{\mathbb{R}}}[0,1]$ is a smooth 1-dimensional manifold equipped with a projection $\pi_{\gamma}^{\text {red }}: \mathbb{R} \mathcal{M}_{\gamma}^{\text {red }} \rightarrow[0,1]$. As soon as $\gamma$ is chosen generic enough, this submanifold intersects $\mathbb{R} \mathcal{M}_{\text {tac }}^{\left(d_{1}, m_{1}\right),\left(d_{2}, m_{2}\right)}$ transversely at finitely many points over distinct parameters of $[0,1]$. Let $C^{t_{0}} \in \mathbb{R} \mathcal{M}_{\gamma}^{\text {red }}$ be such a point, $\left.t_{0} \in\right] 0,1[$. The path $\gamma$ is said to intersect $\pi_{\mathbb{R}}^{\left(d_{1}, m_{1}\right),\left(d_{2}, m_{2}\right)}\left(\mathbb{R} \mathcal{M}_{\text {tac }}^{\left(d_{1}, m_{1}\right),\left(d_{2}, m_{2}\right)}\right)$ positively (resp. negatively) at $\gamma\left(t_{0}\right)$ if $\mathbb{R} \mathcal{M}_{\gamma}^{\text {red }}$ intersects $\mathbb{R} \mathcal{M}_{\text {tac }}^{\left(d_{1}, m_{1}\right),\left(d_{2}, m_{2}\right)}$ positively (resp. negatively) at $C^{t_{0}}$ once endowed with the local orientation induced by $[0,1]$. 
Assume that $\gamma$ is transversal to $\pi_{\mathbb{R}}$ and denote by $\mathbb{R} \mathcal{M}_{\gamma}=\mathbb{R} \mathcal{M}_{\text {cusp }}^{d} \times_{\gamma}[0,1]$. Denote by $\mathbb{R} \overline{\mathcal{M}}_{\gamma}$ the Gromov compactification of $\mathbb{R} \mathcal{M}_{\gamma}$ and by $\pi_{\gamma}: \mathbb{R} \overline{\mathcal{M}}_{\gamma} \rightarrow$ $[0,1]$ the associated projection.

The aim of this subparagraph is to prove the following theorem.

TheOrem 3.2. - Let $\gamma: t \in[0,1] \mapsto\left(J^{t}, \underline{x}^{t}\right) \in \mathbb{R} \mathcal{J}_{\omega} \times \mathbb{R}_{\tau} X^{\nu}$ be a generic path chosen as above and $C^{t_{0}} \in \mathbb{R} \overline{\mathcal{M}}_{\gamma} \cap \mathbb{R} \mathcal{M}_{\text {tac }}^{\left(d_{1}, m_{1}\right),\left(d_{2}, m_{2}\right)}$. Assume that

$$
m_{1}=c_{1}(X) d_{1}-1, \quad m_{2}=c_{1}(X) d_{2}-1,
$$

and that $\gamma$ is positive at $t_{0}=\pi_{\gamma}\left(C^{t_{0}}\right)$. Then, there exist a neighbourhood $W$ of $C^{t_{0}}$ in $\mathbb{R} \overline{\mathcal{M}}_{\gamma}$ and $\epsilon>0$ such that for every $\left.t \in\right] t_{0}-\epsilon, t_{0}\left[, \pi_{\gamma}^{-1}(t) \cap W\right.$ is made of two curves having the same mass, the one of $C^{t_{0}}$, and for every $t \in] t_{0}, t_{0}+\epsilon\left[, \pi_{\gamma}^{-1}(t) \cap W=\varnothing\right.$.

Note that reversing the orientation of $[0,1]$ if necessary, we can always assume that $\gamma$ is positive at $t_{0}$.

Let $C^{t}$ be a real rational cuspidal $J^{t}$-holomorphic curve close to $C^{t_{0}}$ which passes through $\left.\underline{x}^{t}, t \in\right] t_{0}-\epsilon, t_{0}+\epsilon\left[\backslash\left\{t_{0}\right\}\right.$. Then, from Proposition 2.16 of [11], $C^{t}$ extends to a 1-parameter family of $J^{t}$-holomorphic curves $C^{t}(\eta), \eta \in\left[0, \eta_{t}\right.$ [ such that $C^{t}(0)=C^{t}, C^{t}(\eta)$ passes through $\underline{x}^{t}$ for every $\left.\eta \in\right] 0, \eta_{t}\left[\right.$ and $\mathbb{R} C^{t}(\eta)$ has a non isolated real double point in the neighbourhood of the cusp of $C^{t}$ as soon as $\eta \neq 0$.

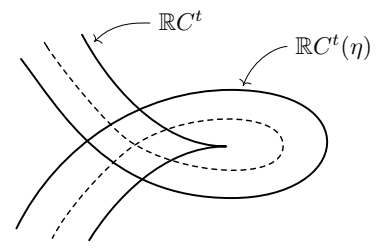

FIGURE 2

LEMMA 3.3. - If $\epsilon$ is small enough, the family $C^{t}(\eta)$ converges to a reducible $J^{t}$-holomorphic curve when $\eta \rightarrow \eta_{t}$.

Note that Lemma 3.3 already implies the second part of Theorem 3.2, since for $t>0$, there are no reducible $J^{t}$-holomorphic curve which pass through $\underline{x}^{t}$ and have a real double point in the neighbourhood of the tacnode of $C^{t_{0}}$.

Proof. - Let $U$ be a compact neighbourhood of $C^{t_{0}}$ in $X$ such that for every $t \in] t_{0}-\epsilon, t_{0}+\epsilon$, the only reducible $J^{t}$-holomorphic curve which pass through $\underline{x}^{t}$ are the ones close to $C^{t_{0}}$. Note that as soon as $\eta$ is close enough to zero, the real parts $\mathbb{R} C^{t}(\eta)$ form a loop around the cusp of $\mathbb{R} C^{t}$ in $\mathbb{R} X$. Moreover, the intersections between two curves of this family $C^{t}(\eta)$ are located at $\underline{x}^{t}$ and in the neighbourhood of their double points. Thus, as $\eta$ grows, the loops grow in 
order to fill a disk of $\mathbb{R} X$ centered at the cusp of $\mathbb{R} C^{t}$. The following alternative now follows from Gromov's compactness Theorem. Either $C^{t}(\eta)$ converges to a reducible $J^{t}$-holomorphic curve in $U$ as $\eta \rightarrow \eta_{t}$, or one curve $C^{t}(\eta)$ intersects the boundary of $U$. Assume that there exists a sequence $\left.t_{n} \in\right] t_{0}-\epsilon, t_{0}+\epsilon\left[\backslash\left\{t_{0}\right\}\right.$, $n \in \mathbb{N}^{*}$, which converges to $t_{0}$ when $n \rightarrow \infty$ and $\eta_{n}>0$ such that $C^{t_{n}}\left(\eta_{n}\right)$ touches the boundary of $U$. Then, when $n \rightarrow \infty, C^{t_{n}}\left(\eta_{n}\right)$ converges to a $J^{t_{0}}$ holomorphic curve $C^{\infty}$ which is contained in $U$, intersects the boundary of $U$ and passes through $\underline{x}^{t_{0}}$. The latter cannot be reducible from the definition of $U$. Moreover, for every $n \in \mathbb{N}^{*}$, the loop of $\mathbb{R} C^{t_{n}}\left(\eta_{n}\right)$ surounds the cusp of $\mathbb{R} C^{t_{n}}$ in $\mathbb{R} X$. It follows that in the limit, $\mathbb{R} C^{\infty}$ forms a loop which surounds the tacnode of $\mathbb{R} C^{t_{0}}$. Thus, $C^{\infty}$ intersects $C^{t_{0}}$ with multiplicity four at least near the tacnode of $C^{t_{0}}$, with multiplicity 2 at least near every double point of $C^{t_{0}}$ and with multiplicity 1 at $\underline{x}^{t_{0}}$. The total intersection index between $C^{\infty}$ and $C^{t_{0}}$ is then at least $d^{2}+2$, which is impossible.

Lemma 3.4. - Assume that $\epsilon$ is small enough and that $t \in] t_{0}-\epsilon, t_{0}[$. Then, the number of cuspidal $J^{t}$-holomorphic curves which pass through $\underline{x}^{t}$ and are close to $C^{t_{0}}$ is at most two.

Proof. - Denote by $C_{\text {red }}^{t}$ the unique reducible real rational $J^{t}$-holomorphic curve which passes through $\underline{x}^{t}$ and is close to $C^{t_{0}}$. This curve has two non isolated real double points $y_{1}^{t}, y_{2}^{t}$ in a neighbourhood of the tacnode of $C^{t_{0}}$. Let $C_{1}^{t}$ be a real rational cuspidal $J^{t}$-holomorphic curve which passes through $\underline{x}^{t}$ and is close to $C^{t_{0}}$ and $C_{1}^{t}(\eta), \eta \in\left[0, \eta_{1}^{t}\right]$, the 1-parameter family of $J^{t}$-holomorphic curves given by Lemma 3.3. In particular, $C_{1}^{t}\left(\eta_{1}^{t}\right)=C_{\text {red }}^{t}$. For every $\left.\eta \in\right] 0, \eta_{1}^{t}[$, denote by $y_{1}^{t}(\eta)$ the real double point of $C_{1}^{t}(\eta)$ close to the tacnode of $C^{t_{0}}$. The latter converges to the cusp of $C_{1}^{t}$ when $\eta \rightarrow 0$ and to one of the points $y_{1}^{t}$, $y_{2}^{t}$, say $y_{1}^{t}$, when $\eta \rightarrow \eta_{1}^{t}$. Assume that there were two families $C_{1}^{t}(\eta), C_{2}^{t}(\delta)$ of curves having this property. Then, for $\eta, \delta$ close to $\eta_{1}^{t}, \delta_{1}^{t}$, the curves $C_{1}^{t}(\eta)$, $C_{2}^{t}(\delta)$ would have all their intersections at $\underline{x}^{t}$ and in the neighbourhood of the double points of $C_{\text {red }}^{t}$ different from $y_{2}^{t}$. Moreover, if $\eta$ is close enough to $\eta_{1}^{t}$, we can assume that the loop formed by $\mathbb{R} \mathbb{C}_{2}^{t}(\delta)$ close to the cusp of $C_{1}^{t}$ is in the interior of the one formed by $\mathbb{R} C_{1}^{t}(\eta)$.

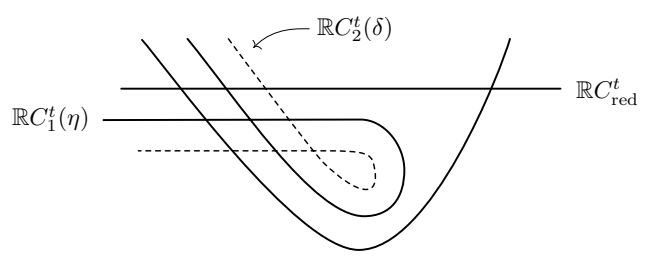

Figure 3

Then, $\mathbb{R} C_{1}^{t}(\eta)$ intersects $\mathbb{R} \mathbb{C}_{2}^{t}(\delta)$ at two points belonging to the two local branches of $\mathbb{R}_{2}^{t}(\delta)$ near $y_{1}^{t}$. As $\eta$ decreases, there is some parameter $\eta^{\prime}$ for

TOME $134-2006-\mathrm{N}^{\mathrm{O}} 2$ 
which $\mathbb{R} C_{1}^{t}\left(\eta^{\prime}\right)$ passes through the double point of $\mathbb{R} \mathbb{C}_{2}^{t}(\delta)$ belonging to its loop. For this parameter $\eta^{\prime}$, the two curves $C_{1}^{t}\left(\eta^{\prime}\right)$ and $\mathbb{C}_{2}^{t}(\delta)$ would have at least three intersection points in the neighbourhood of the tacnode of $C^{t_{0}}$ and thus a total intersection at least equal to $d^{2}+1$, which is impossible. We deduce that the number of real rational cuspidal $J^{t}$-holomorphic curves close to $C^{t_{0}}$ is bounded by the number of real double points of $C_{\text {red }}^{t}$ close to the tacnode of $C^{t_{0}}$, that is two.

Proof of the Theorem 3.2. - It follows from Lemmas 3.3 and 3.4 that there exist a neighbourhood $W$ of $C^{t_{0}}$ in $\mathbb{R} \overline{\mathcal{M}}_{\gamma}$ and $\epsilon>0$ such that for every $t \epsilon$ ]$t_{0}-\epsilon, t_{0}\left[, \#\left(\pi_{\gamma}^{-1}(t) \cap W\right) \leq 2\right.$ and for every $\left.t \in\right] t_{0}, t_{0}+\epsilon\left[, \pi_{\gamma}^{-1}(t) \cap W=\varnothing\right.$. Since the parity of the number of real rational cuspidal $J$-holomorphic curves which pass through $\underline{x}$ does not depend on the generic choice of $(J, \underline{x})$, it suffices to prove that $\pi_{\gamma}^{-1}(t) \cap W$ cannot be empty when $\left.t \in\right] t_{0}-\epsilon, t_{0}[$, that is there exists at least one real rational cuspidal $J$-holomorphic curves which passes through $\underline{x}$ for some $J$ close to $J^{t_{0}}$. Now such a curve can be constructed by reversing the construction of Lemma 3.3. One starts with a reducible real $J$-holomorphic curve having two real non isolated double points close to the tacnode of $C^{t_{0}}$. Then, from Proposition 2.14 of [11], one can smooth one of these double points to obtain a 1-parameter family $\mathbb{R} C(\eta)$ of curves which forms a loop. As $\eta$ decreases, it has to degenerate onto a cuspidal curve by some argument similar to the one used in the proof of Lemma 3.3.

REMARK 3.5. - When $r=(0, \ldots, 0)$, a sequence of real rational cuspidal $J$ holomorphic curves can converge to a reducible curve $C$ having two irreducible components which are complex conjugated and transversal to each other except at one point which is of order two, that is an ordinary tacnode. To extend Theorem 0.1 , one should take into account these reducible curves. This would be possible provided an analog of Theorem 3.2 holds in this case. Namely, assume that over a path $\gamma$, the curve deforms to a reducible curve having two real (resp. complex conjugated) double points in a neighbourhood of the tacnode for $t \in] t_{0}-\epsilon, t_{0}[$ (resp. $t \in] t_{0}, t_{0}+\epsilon[$ ). Then, one can suspect that for $t \in] t_{0}, t_{0}+\epsilon\left[\right.$, there are no real rational cuspidal $J^{t}$-holomorphic curve close to $C$ whereas there are two of them for $t \in] t_{0}-\epsilon, t_{0}[$. Moreover, the latter come from the two degenerations of the figure eight. However, I have no proof of this fact and thus leave this case open.

\subsection{Neighbourhood of $\mathbb{R} \mathcal{M}_{\text {tac }}^{\left(d_{1}, m_{1}\right),\left(d_{2}, m_{2}\right)}$ in $\mathbb{R} \overline{\mathcal{M}}_{\text {cusp }}^{d}$ when $m_{2}=$ $c_{1}(X) d_{2}$}

Let $\gamma: t \in[0,1] \mapsto\left(J^{t}, \underline{x}^{t}\right) \in \mathbb{R} \mathcal{J}_{\omega} \times \mathbb{R}_{\tau} X^{\nu}$ be a generic path transversal to $\pi_{\mathbb{R}}$ and $\mathbb{R} \mathcal{M}_{\gamma}=\mathbb{R} \mathcal{M}_{\text {cusp }}^{d} \times_{\gamma}[0,1]$. Denote by $\mathbb{R} \overline{\mathcal{M}}_{\gamma}$ the Gromov compactification of $\mathbb{R} \mathcal{M}_{\gamma}$ and by $\pi_{\gamma}: \mathbb{R} \overline{\mathcal{M}}_{\gamma} \rightarrow[0,1]$ the associated projection. 
TheOREM 3.6. - Let $\gamma: t \in[0,1] \mapsto\left(J^{t}, \underline{x}^{t}\right) \in \mathbb{R} \mathcal{J}_{\omega} \times \mathbb{R}_{\tau} X^{\nu}$ be a generic path chosen as above and $C^{t_{0}} \in \mathbb{R} \overline{\mathcal{M}}_{\gamma} \cap \mathbb{R} \mathcal{M}_{\mathrm{tac}}^{\left(d_{1}, m_{1}\right),\left(d_{2}, m_{2}\right)}$. Assume that

$$
m_{1}=c_{1}(X) d_{1}-2 \text { and } m_{2}=c_{1}(X) d_{2} .
$$

Then, there exist a neighbourhood $W$ of $C^{t_{0}}$ in $\mathbb{R} \overline{\mathcal{M}}_{\gamma}$ and $\epsilon>0$ such that for every $t \in] t_{0}-\epsilon, t_{0}+\epsilon\left[, \pi_{\gamma}^{-1}(t) \cap W=\left\{C^{t}\right\}\right.$. Moreover, the mass of $C^{t}$ does not depend on $t \in] t_{0}-\epsilon, t_{0}+\epsilon[$.

(See $\S 1.5$ for the definition of the space $\mathbb{R} \mathcal{M}_{\mathrm{tac}}^{\left(d_{1}, m_{1}\right),\left(d_{2}, m_{2}\right)}$.)

Without loss of generality, we can assume that $\underline{x}^{t} \in \mathbb{R}_{\tau} X^{\nu}$ does not depend on $t \in[0,1]$. Denote by $C_{1}^{t_{0}}\left(\operatorname{resp} C_{2}^{t_{0}}\right)$ the irreducible component of $C^{t_{0}}$ which has homology class $d_{1}$ (resp. $d_{2}$ ). Let $\underline{x}_{1}^{t}=\underline{x}^{t} \cap C_{1}^{t_{0}}, \underline{x}_{2}^{t}=\underline{x}^{t} \cap C_{2}^{t_{0}}$ and $U$ be a compact neighbourhood of $C^{t_{0}}$ in $X$. If $U$ is small enough, the curve $C_{1}^{t_{0}}$ extends to a 1 -parameter family $C_{1}^{t_{0}}(\eta), \eta \in[-1,1]$, of real $J^{t_{0}}$-holomorphic curves which pass through $\underline{x}_{1}^{t}$, are contained in $U$ and such that $C_{1}^{t_{0}}(0)=C_{1}^{t_{0}}, C_{1}^{t_{0}}( \pm 1) \cap \partial U \neq \varnothing$ whereas $C_{1}^{t_{0}}(\eta) \subset \stackrel{\circ}{U}$ for $\left.\eta \in\right]-1,1[$. We can assume that the curves $\mathbb{R} C_{1}^{t_{0}}(\eta)$ and $\mathbb{R} C_{2}^{t_{0}}$ have two real intersection points in a neighbourhood of the tacnode of $C^{t_{0}}$ when $\eta>0$. Let $C^{t}$ be a real rational cuspidal $J^{t}$-holomorphic curve close to $C^{t_{0}}$ and which passes through $\left.\underline{x}^{t}, t \in\right] t_{0}-\epsilon, t_{0}+\epsilon\left[\backslash\left\{t_{0}\right\}\right.$. Then, from Proposition 2.16 of [11], $C^{t}$ extends to a 1-parameter family of $J^{t}$-holomorphic curves $C^{t}(\eta), \eta \in[0,1]$ such that $C^{t}(0)=C^{t}, C^{t}(\eta)$ passes through $\underline{x}^{t}$ for every $\eta \in[0,1]$ and $\mathbb{R} C^{t}(\eta)$ has a non isolated real double point in the neighbourhood of the cusp of $C^{t}$ as soon as $\eta \neq 0$. Note that in contrast with $\S 3.1$, as soon as $U$ is small enough, this family cannot break into a reducible curve as long as it stays in $U$. We can thus assume that $C^{t}(1) \cap \partial U \neq \varnothing$.

LEMMA 3.7. - As soon as $U$ is small enough, $C^{t}(1)$ converges to $C^{t_{0}}(1)$ as $t$ converges to $t_{0}$.

Proof. - It suffices to prove that as $t$ converges to $t_{0}$, the curve $C^{t}(1)$ converges to a reducible curve. Indeed, since this curve in the limit is contained in $U$, touches $\partial U$ and passes through $\underline{x}^{t_{0}}$, it has to coincide with $C^{t_{0}}(1)$. Remember that when $t \in] t_{0}-\epsilon, t_{0}+\epsilon\left[\backslash\left\{t_{0}\right\}\right.$, the curve $\mathbb{R} C^{t}(1)$ forms a loop which surrounds the cusp of $\mathbb{R} C^{t}$. Hence, if the curve in the limit were irreducible, its real part would form a loop which would surround the tacnode of $C^{t_{0}}$. As soon as $U$ is small enough, the latter would then intersect $C^{t_{0}}$ with multiplicity 4 near the tacnode, with multiplicity 2 near every double point of $C^{t_{0}}$ and at $\underline{x}^{t_{0}}$, which is impossible.

Hence, the family $\left.\left.C^{t}(\eta), \eta \in\right] 0,1\right]$, is obtained after smoothing one of the two real double points of $C^{t_{0}}(\eta)$ close to the tacnode of $C^{t_{0}}$.

TOME $134-2006-\mathrm{N}^{\mathrm{O}} 2$ 
LemmA 3.8. - Let $C^{t}(\eta)$ and $\left.C^{t}(\eta)^{\prime}, t \in\right] t_{0}-\epsilon, t_{0}+\epsilon\left[\backslash\left\{t_{0}\right\}, \eta \in[0,1]\right.$, be two families of real rational $J^{t}$-holomorphic curves which pass through $\underline{x}^{t}$ and such that $C^{t}(0), C^{t}(0)^{\prime}$ are cuspidal. Then, these families are obtained after smoothing the same real double point of the family $C^{t_{0}}(\eta)$.

Proof. - Assume the converse. Then, the curves $C^{t}(1)$ and $C^{t}\left(\frac{1}{2}\right)^{\prime}$ would intersect (see Figure 4) at $\underline{x}^{t}$ and with multiplicity 2 near every double point of $C^{t_{0}}$. Moreover, the loops formed by $\mathbb{R} C^{t}(1)$ and $\mathbb{R} C^{t}\left(\frac{1}{2}\right)^{\prime}$ would intersect at two points at least. Finally, since $C^{t}\left(\frac{1}{2}\right)^{\prime}$ is not obtained after smoothing the same real double point of the family $C^{t_{0}}(\eta)$ as $C^{t}(1)$, the local real branch in this smoothing which is not included in the loop of $\mathbb{R} C^{t}\left(\frac{1}{2}\right)^{\prime}$ would also intersect $\mathbb{R} C^{t}(1)$ near the tacnode of $C^{t_{0}}$. This would provide a total intersection index at least equal to $\nu+d^{2}-c_{1}(X) d+3>d^{2}$, hence the contradiction.

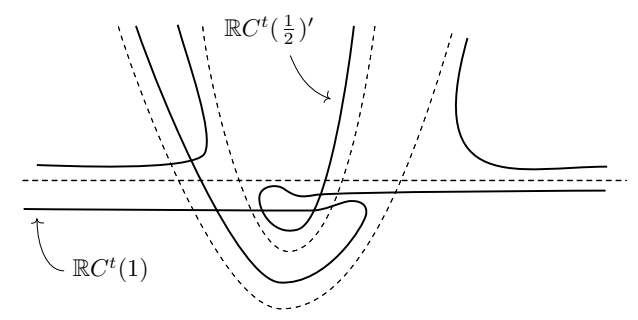

Figure 4

REMARK 3.9. - It also follows from the proof of Lemma 3.8 that if the families $C^{t}(\eta)$ and $C^{t}(\eta)^{\prime}$ are obtained after smoothing the same real double point of the family $C^{t_{0}}(\eta), \eta \in[0,1]$, then the loop formed by $\mathbb{R} C^{t}\left(\frac{1}{2}\right)^{\prime}$ is included in the one formed by $\mathbb{R} C^{t}(1)$. Indeed, these curves would otherwise also intersect with multiplicity at least three near the tacnode of $C^{t_{0}}$, which is impossible.

Proof of Theorem 3.6. - There exist a neighbourhood $W$ of $C^{t_{0}}$ in $\mathbb{R} \overline{\mathcal{M}}_{\gamma}$ and $\epsilon>0$ such that for every $t \in] t_{0}-\epsilon, t_{0}+\epsilon\left[, \#\left(\pi_{\gamma}^{-1}(t) \cap W\right) \leq 1\right.$. Indeed, if this set would contain two curves, they would generate two families $C^{t}(\eta)$ and $C^{t}(\eta)^{\prime}$ as in Lemmas 3.7 and 3.8. From Lemma 3.8, these two families would be obtained after smoothing the same real double point of the family $C^{t_{0}}(\eta)$. From Remark 3.9, the loop formed by $\mathbb{R} C^{t}\left(\frac{1}{2}\right)^{\prime}$ and hence by $\mathbb{R} C^{t}(\eta)^{\prime}$ for every $\eta \in] 0,1]$ would be included in the one of $\mathbb{R} C^{t}(1)$. We then obtain a contradiction repeating the proof of Lemma 3.4. Moreover, as in the proof of Theorem 3.2, $\pi_{\gamma}^{-1}(t) \cap W$ cannot be empty for every $\left.t \in\right] t_{0}-\epsilon, t_{0}+\epsilon[, \epsilon$ small enough. Since the parity of the cardinality of $\pi_{\gamma}^{-1}(t) \cap W$ does not depend on $t$ and the masses of cuspidal curves close to $C^{t_{0}}$ are obviously the ones of $C^{t_{0}}$, the theorem is proved. 


\subsection{Neighbourhood of $\mathbb{R} \mathcal{M}_{\text {cusp }}^{\left(d_{1}, m_{1}\right),\left(d_{2}, m_{2}\right)}$ in $\mathbb{R} \overline{\mathcal{M}}_{\text {cusp }}^{d}$}

Proposition 3.10. - Let $\left(C^{0}, J^{0}\right) \in \mathbb{R} \mathcal{M}_{\text {cusp }}^{\left(d_{1}, m_{1}\right),\left(d_{2}, m_{2}\right)}$. Then, there exists a path $\left(C^{t}, J^{t}\right) \in \mathbb{R} \mathcal{M}_{\text {cusp }}^{\left(d_{1}, m_{1}\right),\left(d_{2}, m_{2}\right)}, t \in[0,1]$, of class $C^{\ell-k}$, such that $J^{1}$ is integrable in a neighbourhood of $C^{1}$ in $X$.

(See $\S 1.5$ for the definition of the space $\mathbb{R} \mathcal{M}_{\text {cusp }}^{\left(d_{1}, m_{1}\right),\left(d_{2}, m_{2}\right)}$.)

LEMmA 3.11. - Under the hypothesis of Proposition 3.10, there exists a neighbourhood $V$ of the singular points of $C^{0}$ in $X$ and a path $\left(C^{t}, J^{t}\right) \in$ $\mathbb{R} \mathcal{M}_{\text {cusp }}^{\left(d_{1}, m_{1}\right),\left(d_{2}, m_{2}\right)}, t \in[0,1]$, of class $C^{\ell-k}$, such that $J^{1} \mid V$ is integrable.

Proof. - Let $y_{0}$ be a double point of $C^{0}$. Then, there exists a neighbourhood $V_{0}$ of $y_{0}$ in $X$, invariant under $c_{X}$, as well as a diffeomorphism $\Phi: V_{0} \rightarrow B^{4}$ which is $\mathbb{Z} / 2 \mathbb{Z}$-equivariant such that

$$
\Phi\left(C^{0} \cap V_{0}\right)=\left\{\left(w_{1}, w_{2}\right) \in B^{4} \subset \mathbb{C}^{2} \mid w_{1} w_{2}=0\right\} .
$$

Denote by $J$ the almost complex structure $\Phi_{*}\left(J^{0} \mid V_{0}\right)$ of $B^{4}$, and for $\left.\left.\delta \in\right] 0,1\right]$, by $h_{\delta}$ the homothety $\left(w_{1}, w_{2}\right) \in B^{4} \mapsto\left(\delta w_{1}, \delta w_{2}\right) \in B^{4}$. Set then $J_{1-\delta}=h_{\delta}^{*}(J)$, so that for $\xi \in \mathbb{R}^{4}=T_{\left(w_{1}, w_{2}\right)} B^{4}$,

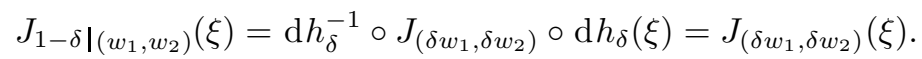

Hence, the family $\left(J_{1-\delta}\right)_{\delta \in] 0,1]}$, extend to a $C^{\infty}$-family $\left(J_{\delta}\right)_{\delta \in[0,1]}$, by setting $J_{1} \equiv J_{(0,0)}$. Now, $\Phi\left(C^{0} \cap V_{0}\right)$ is invariant under $h_{\delta}$ and thus $J_{\delta}$-holomorphic for every $\delta \in[0,1]$. The family $\left(\Phi^{*} J_{\delta}\right)_{\delta \in[0,1]}$ is then a path of almost complex structures of class $C^{\ell}$ of $V_{0}$ such that $\Phi^{*} J_{0}=J^{0} \mid V_{0}, C^{0} \cap V_{0}$ is $\Phi^{*} J_{\delta^{-}}$ holomorphic for every $\delta \in[0,1]$ and $\Phi^{*} J_{1}$ is integrable. There is no obstruction to extend $\left(\Phi^{*} J_{\delta}\right)_{\delta \in[0,1]}$ to a path of almost complex structures of class $C^{\ell}$ on the whole $X$ for which $C^{0}$ is holomorphic and which coincide with $J^{0}$ outside a neighbourhood of $V_{0}$. Now, if $y_{0}$ is the unique real ordinary cusp of $C^{0}$, we can proceed in the same way, making use of the weighted homothety $\tilde{h}_{\delta}:\left(w_{1}, w_{2}\right) \in B^{4} \mapsto\left(\delta^{2} w_{1}, \delta^{3} w_{2}\right) \in B^{4}$ instead of $h_{\delta}$. The proof is then the same as the one of Lemma 2.6 of [11] and is not reproduced here.

Proof of Proposition 3.10. - From Lemma 3.11, we can assume that $J^{0}$ is integrable in a neighbourhood $V_{0}$ of the singular points of $C^{0}$. Let $\bar{C}_{0} \subset C^{0}$ be a smooth compact curve with boundary such that $C^{0} \backslash \bar{C}_{0} \subset V_{0}$. A tubular neighbourhood $N$ of $\bar{C}_{0}$ in $X$ is identified with a neighbourhood of the zero section in the normal bundle of $\bar{C}_{0}$ in $X$. Denote by $p: N \rightarrow \bar{C}_{0}$ the projection induced by this identification and equip $N$ with the almost complex structure $J_{\mid N}$. This identification can be chosen such that the fibres of $p$ are

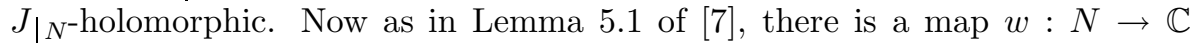
which is holomorphic and injective once restricted to each fibre of $p$. Such a map can be constructed as follows. Extend $J_{\mid N}$ to an almost complex structure

TOME $134-2006-\mathrm{N}^{\mathrm{O}} 2$ 
on the whole compactified normal bundle $\bar{N}$ over $C^{0}$, such that $p: \bar{N} \rightarrow C^{0}$ is a sphere bundle with $J$-holomorphic fibres, the section $C^{\infty}$ at infinity is $J$ holomorphic and a third section $C^{1}$ distinct from $C^{0}$ and $C^{\infty}$ is $J$-holomorphic. The function $w: \bar{N} \rightarrow \mathbb{C} P^{1}$ is then the unique one given by Riemann's uniformization theorem which is holomorphic once restricted to each fibre and sends $C^{0}, C^{\infty}$ and $C^{1}$ to $0, \infty$ and 1 respectively. Let $z: \bar{C}_{0} \rightarrow \mathbb{C}$ be an injective holomorphic map, the composition with $p$ will also be denoted by $z: N \rightarrow \mathbb{C}$. The antiholomorphic tangent bundle $T_{N, J \mid N}^{0,1}$ is then generated by $\partial_{\bar{w}}$ and $\partial_{\bar{z}}+a \partial_{z}+b \partial_{w}$. Moreover, $J_{\mid N}$ is integrable if and only if $\partial_{\bar{w}} a=0=\partial_{\bar{w}} b$ (see [7], Lemma 1.3). This is in particular the case on $N \cap V_{0}$. Let $f: \bar{C}_{0} \rightarrow \mathbb{R}$ be a $C^{\infty}$ function which is equal to 1 in a neighbourhood of the boundary of $\bar{C}_{0}$ and to 0 on $\bar{C}_{0} \backslash V_{0}$. For $\delta \in[0,1]$, denote by $J_{\delta}$ the almost complex structure on $N$ whose antiholomorphic tangent bundle $T_{N, J_{\delta} \mid N}^{0,1}$ is generated by $\partial_{\bar{w}}$ and $\partial_{\bar{z}}+((1-\delta)+\delta f \circ p)\left(a \partial_{z}+b \partial_{w}\right)$. Then, $J_{0}=J_{\mid N}$ and $J_{1}$ is integrable since

$$
\partial_{\bar{w}}(f \circ p a)=f \circ p \partial_{\bar{w}} a=0 \quad \text { and } \quad \partial_{\bar{w}}(f \circ p b)=f \circ p \partial_{\bar{w}} b=0 .
$$

Hence the result.

Let $\gamma: t \in[0,1] \mapsto\left(J^{t}, \underline{x}^{t}\right) \in \mathbb{R}_{\mathcal{\omega}} \times \mathbb{R}_{\tau} X^{\nu}$ be a path transversal to $\pi_{\mathbb{R}}$ and

$$
\pi_{\mathbb{R}}^{\left(d_{1}, m_{1}\right),\left(d_{2}, m_{2}\right)}: \mathbb{R} \mathcal{M}_{\text {cusp }}^{\left(d_{1}, m_{1}\right),\left(d_{2}, m_{2}\right)} \longrightarrow \mathbb{R} \mathcal{J}_{\omega} \times \mathbb{R}_{\tau_{1}} X^{m_{1}} \times \mathbb{R}_{\tau_{2}} X^{m_{2}} .
$$

Let $\mathbb{R} \mathcal{M}_{\gamma}=\mathbb{R} \mathcal{M}_{\text {cusp }}^{d} \times_{\gamma}[0,1], \mathbb{R} \overline{\mathcal{M}}_{\gamma}$ its Gromov compactification and $\pi_{\gamma}$ : $\mathbb{R} \overline{\mathcal{M}}_{\gamma} \rightarrow[0,1]$ the associated projection.

Theorem 3.12. - Let $\gamma: t \in[0,1] \mapsto\left(J^{t}, \underline{x}^{t}\right) \in \mathbb{R} \mathcal{J}_{\omega} \times \mathbb{R}_{\tau} X^{\nu}$ be a generic path chosen as above and $C^{t_{0}} \in \mathbb{R} \overline{\mathcal{M}}_{\gamma} \cap \mathbb{R} \mathcal{M}_{\text {cusp }}^{\left(d_{1}, m_{1}\right),\left(d_{2}, m_{2}\right)}$. Then, there exist a neighbourhood $W$ of $C^{t_{0}}$ in $\mathbb{R} \overline{\mathcal{M}}_{\gamma}$ and $\epsilon>0$ such that $\# \pi_{\gamma}^{-1}(t) \cap W$ does not depend on the choice of $t \in] t_{0}-\epsilon, t_{0}+\epsilon\left[\backslash\left\{t_{0}\right\}\right.$.

Note that as soon as $W$ is small enough, all the curves of $W$ have the same mass, the one of $C^{t_{0}}$.

LEMMA 3.13. - Under the hypothesis of Theorem 3.12, we can assume that $\underline{x}^{t}$ does not depend on $\left.t \in\right] t_{0}-\epsilon, t_{0}+\epsilon\left[\right.$ and that $\left(J^{t}\right)_{t \in] t_{0}-\epsilon, t_{0}+\epsilon[}$ is an analytic path of almost complex structures which are integrable in a neighbourhood of $C^{t_{0}}$ in $X$.

Proof. - From Proposition 3.10, we can assume that $J^{t_{0}}$ is integrable in a neighbourhood of $C^{t_{0}}$. Indeed, let $J^{t_{0}}(\lambda), \lambda \in[0,1]$, be the path in $\mathbb{R} \mathcal{M}_{\text {cusp }}^{\left(d_{1}, m_{1}\right),\left(d_{2}, m_{2}\right)}$ given by this proposition, such that $J^{t_{0}}(0)=J^{t_{0}}$ and $J^{t_{0}}(1)$ is integrable in a neighbourhood of $C^{t_{0}}$. There is no obstruction to extend this path in a 2-parameters family $\left.J^{t}(\lambda), \lambda \in[0,1], t \in\right] t_{0}-\epsilon, t_{0}+\epsilon[$, such that $J^{t}(0)=J^{t}$ and $\left(J^{t}(\lambda), \underline{x}^{t}\right)$ satisfies the hypothesis of Theorem 3.12 for every $\lambda \in[0,1]$. From Lemma 1.5 and the definition of $\mathbb{R} \mathcal{M}_{\text {cusp }}^{\left(d_{1}, m_{1}\right),\left(d_{2}, m_{2}\right)}$, none of 
the elements of $\pi_{\gamma}^{-1}(t)$ can degenerate onto a critical point of $\pi_{\mathbb{R}}$ or a reducible curve over $J^{t}(\lambda)$. Thus, the cardinality of $\pi_{\mathbb{R}}^{-1}\left(J^{t}(\lambda), \underline{x}^{t}\right) \cap W(\lambda)$, where $W(\lambda)$ is a neighbourhood of $C^{t_{0}}(\lambda)$ in $\mathbb{R} \overline{\mathcal{M}}_{\text {cusp }}^{d}$, does not depend on $\lambda \in[0,1]$.

We have to prove that a transversal path $\left(J^{t}\right)_{t \in] t_{0}-\epsilon, t_{0}+\epsilon[}$ to $\mathbb{R} \mathcal{M}_{\text {cusp }}^{\left(d_{1}, m_{1}\right),\left(d_{2}, m_{2}\right)}$ can be chosen analytic and made of almost complex structures which are integrable in a neighbourhood of $C^{t_{0}}$ in $X$. Assume that $m_{1}=c_{1}(X) d_{1}-1$, and denote by $C_{1}^{t_{0}}=\left[u_{1}, J_{S}^{1}, J^{t_{0}}, \underline{x}_{1}, z_{c}\right]$ the cuspidal component of $C^{t_{0}}$, so that $\underline{x}_{1}=\underline{x}^{t_{0}} \cap C_{1}^{t_{0}}$ is of cardinality $m_{1}$. Then, from Proposition 1.9 , the path $\left(J^{t}\right)_{t \in] t_{0}-\epsilon, t_{0}+\epsilon[}$ is transversal to $\mathbb{R} \mathcal{M}_{\text {cusp }}^{\left(d_{1}, m_{1}\right),\left(d_{2}, m_{2}\right)}$ at $J^{t_{0}}$ if and only if $\dot{J}^{t_{0}}=\frac{\mathrm{d}}{\mathrm{d} t} J_{\mid t=t_{0}}$ is such that the $J_{S}^{1}$ antilinear form $\dot{J}^{t_{0}} \circ \mathrm{d} u_{1} \circ J_{S}^{1}$ projects onto a generator of the cokernel $H_{D}^{1}\left(S ; \mathcal{N}_{u_{1},-z_{1}}^{\text {cusp }}\right)_{+1} \cong \mathbb{R}$. Let $\alpha$ be a generator of $H_{D}^{1}\left(S ; \mathcal{N}_{u_{1},-z_{1}}^{\text {cusp }}\right)_{+1}$ having support in a small ball $U$ of $S$. Let $V$ be an open subset of $\left(X, J^{t_{0}}\right)$ biholomorphic to the bidisc $\bar{B}^{2} \times \bar{B}^{2} \subset \mathbb{C}^{2}$ and such that $V \cap C^{t_{0}}=u_{1}(U)$. We choose this biholomorphism such that it sends $V \cap C^{t_{0}}$ onto the disc $\{w=0\} \subset \bar{B}^{2} \times \bar{B}^{2}$, where the latter is equipped with complex coordinates $(z, w)$. In this chart, the generator $\alpha$ writes $f(z, \bar{z}) \mathrm{d} \bar{z} \otimes w$, where $f: \bar{B}^{2} \rightarrow \mathbb{C}$ is $\mathbb{Z} / 2 \mathbb{Z}$-equivariant, with compact support and can be assumed to be smooth. For every $t \in] t_{0}-\epsilon, t_{0}+\epsilon\left[\right.$, define then $J_{\mid V}^{t}$ to be the endomorphism given by the matrix

$$
\left[\begin{array}{cc}
i & 0 \\
\left(t-t_{0}\right) f \mathrm{~d} \bar{z} & i
\end{array}\right]
$$

Since $f$ is with compact support, $J^{t}\left|V=J^{t_{0}}\right| V$ in a neighbourhood of the boundary of $V$. We can then extend $J^{t}$ on the whole $X$ by setting $J^{t} \equiv J^{t_{0}}$ outside $V$. The path $t \in] t_{0}-\epsilon, t_{0}+\epsilon\left[\mapsto J^{t}\right.$ is analytic. Moreover, the antiholomorphic complexified tangent bundle of $\left(X, J^{t}\right)$ is generated by the vectors $\left\langle\partial_{\bar{z}}+\frac{1}{2}\left(t-t_{0}\right) f \partial_{w}, \partial_{\bar{w}}\right\rangle$. Since $\frac{1}{2}\left(t-t_{0}\right) f$ does not depend on $w$, it follows from Lemma 1.3 of [7] that $J^{t}$ is integrable on $V$ for every $\left.t \in\right] t_{0}-\epsilon, t_{0}+\epsilon[$. The lemma is proved in the case $m_{1}=c_{1}(X) d_{1}-1$ and can be proved along the same lines when $m_{1}=c_{1}(X) d_{1}-2$.

Proof of Theorem 3.12. - Denote by $B^{2}\left(t_{0}, \epsilon\right)=\left\{t \in \mathbb{C} ;\left|t-t_{0}\right|<\epsilon\right\}$. The path $\gamma: t \in] t_{0}-\epsilon, t_{0}+\epsilon\left[\mapsto J^{t} \in \mathbb{R} \mathcal{J}_{\omega}\right.$ given by Lemma 3.13 is complexified to an analytic path $\gamma_{\mathbb{C}}: t \in B^{2}\left(t_{0}, \epsilon\right) \mapsto J^{t} \in \mathcal{J}_{\omega}$ which is $\mathbb{Z} / 2 \mathbb{Z}$-equivariant and made of almost complex structures which are integrable in a neighbourhood of $C^{t_{0}}$. Equip the product $Y=B^{2}\left(t_{0}, \epsilon\right) \times X$ with the almost complex structure $J_{Y}$ defined by the matrix $\left[\begin{array}{cc}i & 0 \\ 0 & J^{t}\end{array}\right]$. It is integrable in a neighbourhood of $\left\{t_{0}\right\} \times C^{t_{0}}$. Moreover, the sections $\left(t, \underline{x}^{t}\right)$ are $J_{Y}$-holomorphic. Note that the complexified moduli space $\mathcal{M}_{\gamma_{\mathbb{C}}}$ is then a smooth curve which is equipped with a holomorphic projection $\pi: \mathcal{M}_{\gamma_{\mathbb{C}}} \rightarrow B^{2}\left(t_{0}, \epsilon\right) \backslash\left\{t_{0}\right\}$. The complex structure

TOME $134-2006-\mathrm{N}^{\mathrm{O}} 2$ 
of $\mathcal{M}_{\gamma_{\mathrm{C}}}$ extends in a unique way on the compactification $\overline{\mathcal{M}}_{\gamma_{\mathrm{C}}}$ and the projection $\pi: \overline{\mathcal{M}}_{\gamma_{\mathbb{C}}} \rightarrow B^{2}\left(t_{0}, \epsilon\right)$ is holomorphic. We will prove that once restricted to any irreducible component of $\overline{\mathcal{M}}_{\gamma_{\mathrm{c}}}$, this projection is a biholomorphism. From now on, we can assume that $\overline{\mathcal{M}}_{\gamma_{\mathrm{C}}}$ is irreducible. Let $U \rightarrow \overline{\mathcal{M}}_{\gamma_{\mathrm{C}}}$ be the universal curve and $\bar{U}$ be the stable map compactification of $U$. The latter is a complex surface and the projection $\bar{U} \rightarrow \overline{\mathcal{M}}_{\gamma_{\mathrm{C}}}$ is a projective line bundle having a singular fibre over $C^{t_{0}} \in \overline{\mathcal{M}}_{\gamma_{\mathbb{C}}}$. Denote by $\sigma_{1}, \ldots, \sigma_{c_{1}(X) d-2}: \overline{\mathcal{M}}_{\gamma_{\mathbb{C}}} \rightarrow \bar{U}$ the sections associated to the marked points $z_{1}, \ldots, z_{c_{1}(X) d-2}$ of $S$ and by eval $: \bar{U} \rightarrow Y$ the evaluation map, so that for every $C \in \overline{\mathcal{M}}_{\gamma_{\mathrm{C}}}$,

$$
\operatorname{eval}\left(\sigma_{j}(C)\right)=\left(\pi_{\gamma_{\mathrm{C}}}(C), x_{j}^{\pi_{\gamma_{\mathrm{C}}}(C)}\right) \in Y
$$

Denote by $S_{1}, S_{2}$ the two irreducible components of the singular fibre of $\bar{U}$, in such a way that eval $\left(S_{1}\right)$ is the cuspidal component $C_{1}^{t_{0}}$ of $C^{t_{0}}$. Note that since the two components of $C^{t_{0}}$ intersect transversely, $\mathrm{d}_{y}$ eval is injective at the intersection point $y$ of $S_{1} \cap S_{2}$. The normal bundle of $S_{1}$ in $\bar{U}$ is isomorphic to $\mathcal{O}_{S_{1}}(-1)$ and the evaluation map induces a non vanishing morphism from this bundle to the normal bundle of $C_{1}^{t_{0}}$ in $Y$, which is isomorphic to $\mathcal{O}_{S_{1}}\left(c_{1}(X) d_{1}-3\right) \oplus \mathcal{O}_{S_{1}}$. Would the projection $\pi: \overline{\mathcal{M}}_{\gamma_{\mathbb{C}}} \rightarrow B^{2}\left(t_{0}, \epsilon\right)$ not be biholomorphic, this morphism would vanish on $\underline{z}_{1}=\underline{z} \cap S_{1}$ which is of cardinality $m_{1}$. Assume that $m_{1}=c_{1}(X) d_{1}-1$. The image of $\mathcal{O}_{S_{1}}(-1)$ would then be a subline bundle of degree at least $c_{1}(X) d_{1}-2$ of $\mathcal{O}_{S_{1}}\left(c_{1}(X) d_{1}-3\right) \oplus \mathcal{O}_{S_{1}}$. This is impossible. In the same way, if $m_{1}=c_{1}(X) d_{1}-2$, then the normal bundle of $C_{2}^{t_{0}}$ in $Y$ (resp. $\left.\bar{U}\right)$ is isomorphic to $\mathcal{O}_{S_{2}}\left(c_{1}(X) d_{1}-2\right) \oplus \mathcal{O}_{S_{2}}\left(\right.$ resp. $\mathcal{O}_{S_{2}}(-1)$ ). Would the projection $\pi: \overline{\mathcal{M}}_{\gamma_{\mathrm{C}}} \rightarrow B^{2}\left(t_{0}, \epsilon\right)$ not be biholomorphic, the morphism $\mathcal{O}_{S_{2}}(-1) \rightarrow \mathcal{O}_{S_{2}}\left(c_{1}(X) d_{1}-2\right) \oplus \mathcal{O}_{S_{2}}$ would vanish on $\underline{z}_{2}=\underline{z} \cap S_{2}$ which is of cardinality $m_{2}$. Since the latter is equal to $c_{1}(X) d_{2}$, we conclude as before.

\section{Proof of Theorem 0.1}

Let $\left(J^{0}, \underline{x}^{0}\right)$ and $\left(J^{1}, \underline{x}^{1}\right)$ be two regular values of

$$
\pi_{\mathbb{R}}: \mathbb{R} \mathcal{M}_{\text {cusp }}^{d} \longrightarrow \mathbb{R} \mathcal{J}_{\omega} \times \mathbb{R}_{\tau} X^{\nu}
$$

which do not belong to $\pi_{\mathbb{R}}\left(\mathbb{R} \overline{\mathcal{M}}_{\text {cusp }}^{d} \backslash \mathbb{R} \mathcal{M}_{\text {cusp }}^{d}\right)$. We can assume that every real rational cuspidal $J^{i}$-holomorphic curve which pass through $\underline{x}^{i}, i \in\{0,1\}$, and realize the homology class $d$ has a unique real ordinary cusp and transversal double points as singularities, all of them being outside $\underline{x}^{i}$. We have to prove that $\Gamma_{r}^{d}\left(J^{0}, \underline{x}^{0}\right)=\Gamma_{r}^{d}\left(J^{1}, \underline{x}^{1}\right)$.

4.1. Choice of the path $\gamma$. - Let $\gamma: t \in[0,1] \mapsto\left(J^{t}, \underline{x}^{t}\right) \in \mathbb{R} \mathcal{J}_{\omega} \times \mathbb{R}_{\tau} X^{\nu}$ be a generic path transversal to $\pi_{\mathbb{R}}$ joining $\left(J^{0}, \underline{x}^{0}\right)$ to $\left(J^{1}, \underline{x}^{1}\right)$. Denote by

$$
\mathbb{R} \mathcal{M}_{\gamma}=\mathbb{R} \mathcal{M}_{\text {cusp }}^{d} \times_{\gamma}[0,1],
$$

BULLETIN DE LA SOCIÉtÉ MATHÉMATIQUE DE FRANCE 
$\mathbb{R} \overline{\mathcal{M}}_{\gamma}$ its Gromov compactification and $\pi_{\gamma}: \mathbb{R} \overline{\mathcal{M}}_{\gamma} \rightarrow[0,1]$ the associated projection. From Lemma 1.6 and Corollary 1.12, the path $\gamma$ can be chosen such that all elements of $\mathbb{R} \overline{\mathcal{M}}_{\gamma}$ are irreducible real rational curves having a unique real ordinary cusp and only transversal double points as singularities, all of them being outside $\underline{x}^{t}$, with the exception of finitely many of them which may have:

1) A unique real ordinary triple point or a unique real ordinary tacnode.

2) A real branch which crosses the real ordinary cusp transversely to its tangent line.

3) A unique real double point belonging to $\underline{x}^{t}$.

4) The unique real ordinary cusp belonging to $\underline{x}^{t}$.

$5)$ A second real ordinary cusp outside $\underline{x}^{t}$. In this case, any generator $\psi$ of

$$
H^{0}\left(S, K_{S} \otimes N_{u,-\underline{z}}^{*}\right)_{-1} \cong H^{1}\left(S, N_{u,-\underline{z}}\right)_{+1}^{*}
$$

does not vanish at cusps (compare Proposition 2.8 of [11]).

6) A unique cuspidal point, which is a generic order two degenerated cusp, see $\S 2.1$ for a definition.

7) Two irreducible components.

8) Two irreducible components which intersect transversely except at one point which is a real ordinary tacnode. These curves are not cuspidal.

In the same way, the path $\gamma$ can be chosen such that every real rational reducible $J^{t}$-holomorphic curves which pass through $\underline{x}^{t}$ and realize the homology class $d$ have only two irreducible components, both real, and transversal double points as singularities, with the exception of finitely many of them which may have:

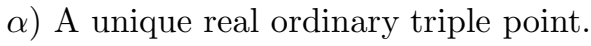

$\beta$ ) A unique real ordinary tacnode.

$\gamma)$ A unique real ordinary cusp. In this case, any generator $\psi$ of

$$
H^{0}\left(S_{1}, K_{S} \otimes N_{u_{1},-\underline{z}_{1}}^{*}\right)_{-1} \cong H^{1}\left(S_{1}, N_{u_{1},-\underline{z}_{1}}\right)_{+1}^{*}
$$

does not vanish at the cusp, where $u_{1}: S_{1} \rightarrow X$ parameterize the cuspidal component (compare Proposition 2.8 of [11]).

$\delta)$ A unique real double point belonging to $\underline{x}^{t}$.

$\epsilon)$ Three irreducible components.

Finally, the path $\gamma$ can be chosen such that every real rational $J^{t}$ holomorphic curve which pass through $\underline{x}^{t}$, realize the homology class $d$, and have one of the lines $T_{i}, i \in I$, as a tangent line at $x_{i}^{t}$, have only transversal double points as singularities, with the exception of finitely many which may have:

a) A unique real ordinary triple point.

b) A unique real ordinary tacnode.

TOME $134-2006-\mathrm{N}^{\mathrm{O}} 2$ 
c) A unique real ordinary cusp outside $\underline{x}^{t}$. In this case, any generator $\psi$ of

$$
H^{0}\left(S, K_{S} \otimes N_{u,-\underline{z}}^{*}\right)_{-1} \cong H^{1}\left(S, N_{u,-\underline{z}}\right)_{+1}^{*}
$$

does not vanish at the cusp (compare Proposition 2.8 of [11]).

d) A unique real ordinary cusp at one point $x_{i}^{t}, i \in I$. In this case, the curve is not tangent to any line $T_{j}, j \in I$. Moreover, any generator $\psi$ of

$$
H^{0}\left(S, K_{S} \otimes N_{u,-\underline{z}}^{*}\right)_{-1} \cong H^{1}\left(S, N_{u,-\underline{z}}\right)_{+1}^{*}
$$

does not vanish at the cusp (compare Proposition 2.8 of [11]).

e) A unique real double point belonging to $\underline{x}^{t}$, none of the branches being tangent to $T_{i}$.

f) Two irreducible components.

g) Two irreducible components which intersect at one point $x_{i}^{t}, i \in I$. In this case, the curve is not tangent to any line $T_{j}, j \in I$.

4.2. The case $\boldsymbol{z}_{\boldsymbol{c}} \in \underline{\boldsymbol{z}}$. - Let $(J, \underline{x}) \in \mathbb{R} \mathcal{J}_{\omega} \times \mathbb{R}_{\tau} X^{\nu}$ be a generic critical value of $\pi_{\mathbb{R}}$ of type 3 given by Lemmas 1.5 and 1.6. Let $C$ be a real rational $J$-holomorphic curve which pass through $\underline{x}$, realize the homology class $d$ and has a unique real ordinary cusp in $\underline{x}$, say at $x_{1}$. Then, the space of real rational cuspidal $J$-holomorphic curves which pass through $\underline{x} \backslash\left\{x_{1}\right\}$ and realize $d$ is a 1parameter family generated by any non zero element of $H^{0}\left(S, N_{u,-\underline{z}+z_{1}}\right)_{+1} \cong \mathbb{R}$. In particular, the cuspidal point moves along a smooth curve $\ell_{c} \subset \mathbb{R} X$ transversal to the tangent line of $\mathbb{R} C$ at the cusp $x_{1}$. Let $U$ be a neighbourhood of $x_{1}$ in $\mathbb{R} X$ diffeomorphic to a ball and small enough so that $\ell_{c}$ divides it in two components. Denote by $U_{-}$(resp. $\left.U_{+}\right)$the connected component of $U \backslash \ell_{c}$ defined by the relation $U_{-} \cap \mathbb{R} C \neq \varnothing\left(\right.$ resp. $\left.U_{+} \cap \mathbb{R} C=\varnothing\right)$.

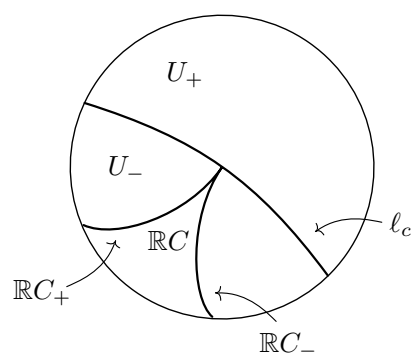

FiguRe 5

Proposition 4.1. - Let $y \in U_{-}$(resp. $y \in U_{+}$). Then, as soon as $U$ is small enough,

1) There are exactly 2 (resp. 0) real rational cuspidal J-holomorphic curves which pass through $\underline{x} \cup\{y\} \backslash\left\{x_{1}\right\}$ and are close to $C$.

BULLETIN DE LA SOCIÉTÉ MATHÉMATIQUE DE FRANCE 
2) There is exactly one real rational J-holomorphic curves close to $C$ which pass through $\underline{x} \cup\{y\} \backslash\left\{x_{1}\right\}$ and has a real double point at $y$. Moreover, this double point is real and non isolated (resp. isolated).

Proof. - As soon as $U$ is small enough, the intersection of $\mathbb{R} C$ with $U_{-}$is made of two arcs denoted by $\mathbb{R} C_{+}$and $\mathbb{R} C_{-}$. The 1-parameter family of real rational cuspidal $J$-holomorphic curves which pass through $\underline{x} \backslash\left\{x_{1}\right\}$ and realize $d$ produces 1 -parameter families of arcs $\mathbb{R} C_{+}$and $\mathbb{R} C_{-}$. As soon as $U$ is small enough, these two families provide two foliations of $U_{-}$. If $y \in U_{-}$, there is exactly one leaf of each foliation which pass through $y$, hence the first part of the proposition in this case. If $y \in U_{+}$, the first part of the proposition follows from the relation $U_{+} \cap \mathbb{R} C=\varnothing$. Let us prove now the second part of the proposition. First, if such a curve exists, it has to be unique. Indeed, two such curves would intersect twice near each double point of $C$, with multiplicity one at each point of $\underline{x} \backslash\left\{x_{1}\right\}$ and with multiplicity four at $y$. Then, their intersection index would be at least

$$
d^{2}-c_{1}(X) d+c_{1}(X) d-3+4=d^{2}+1,
$$

which is impossible. Let $x_{1}^{\prime} \in \mathbb{R} C \cap U_{-}$. From Proposition 2.16 of [11], the curve $\mathbb{R} C$ extends to a 1 -parameter family of real rational $J$-holomorphic curves $\mathbb{R} C(\eta), \eta \in[-1,1]$, which pass through $\underline{x} \cup\left\{x_{1}^{\prime}\right\} \backslash\left\{x_{1}\right\}$ and have a non isolated (resp. isolated) real ordinary double point close to the cusp of $\mathbb{R} C$ when $\eta>0$ (resp. $\eta<0$ ). In the same way as in $\S 3.1$, the curves $\mathbb{R} C(\eta), \eta>0$, form a loop in a neighbourhood of the cusp of $\mathbb{R} C$. Moreover, this 1-parameter family of loops foliates some disk of $\mathbb{R} X$ centered at $x_{1}$. The curve $\ell_{c}$ intersects transversely these loops. Then, the non isolated real double point of $\mathbb{R} C(\eta)$, $\eta>0$, has to be in the same connected component of $U \backslash \ell_{c}$ as the branches $\mathbb{R} C_{+}$and $\mathbb{R} C_{-}$, that is $U_{-}$. Thus, there exists at least one point $y \in U_{-}$for which there is a real rational $J$-holomorphic curve close to $C$ which passes through $\underline{x} \cup\{y\} \backslash\left\{x_{1}\right\}$ and has a non isolated real double point at $y$. By deforming $y \in U_{-}$, we get the same result for all $y \in U_{-}$as soon as $U$ is small enough. The curves $\mathbb{R} C(\eta), \eta<0$, must then have their isolated real double point in $U_{+}$, which proves the result.

Let us assume now that $(J, \underline{x})=\gamma\left(t_{0}\right)$, where $\gamma$ is the path chosen in $\S 4.1$ and $\left.t_{0} \in\right] 0,1[$. Without loss of generality, we can assume that there exists $\epsilon>0$ such that for every $t \in] t_{0}-\epsilon, t_{0}+\epsilon\left[, J^{t} \equiv J^{t_{0}}\right.$ and $\underline{x}^{t} \backslash\left\{x_{1}^{t}\right\} \equiv \underline{x} \backslash\left\{x_{1}\right\}$. The path $\left(x_{1}^{t}\right)_{t \in] t_{0}-\epsilon, t_{0}+\epsilon[}$ is then transverse to $\ell_{c}$ in $\mathbb{R} X$ at $t=t_{0}$.

Proposition 4.2. - The integer $\Gamma_{r}^{d}\left(J^{t}, \underline{x}^{t}\right)$ does not depend on $\left.t \in\right] t_{0}-\epsilon$, $t_{0}+\epsilon\left[\backslash\left\{t_{0}\right\}\right.$.

Proof. - We can assume that for $t \in] t_{0}-\epsilon, t_{0}[$ (resp. $t \in] t_{0}, t_{0}+\epsilon\left[\right.$ ), $x_{1}^{t} \in U_{-}$ (resp. $x_{1}^{t} \in U_{+}$). From the first part of Proposition 4.1, the first term in the 
expression of $\Gamma_{r}^{d}\left(J^{t}, \underline{x}^{t}\right)$ decreases of $2(-1)^{m\left(C^{t}\right)}$ as $t$ crosses the value $t_{0}$. Let us prove that at the same time, the third term in the expression of $\Gamma_{r}^{d}\left(J^{t}, \underline{x}^{t}\right)$ increases of $2(-1)^{m\left(C^{t_{0}}\right)}$ as $t$ crosses the value $t_{0}$. From Proposition 3.4 of [11], we have

$$
\begin{gathered}
\chi_{r+2}^{d}=\sum_{\substack{C \in \mathcal{T a n}^{d}\left(J^{t}, \underline{x}^{t}\right) \\
T_{x_{1}^{t}} \mathbb{R} C=\underline{T}_{1}}}(-1)^{m(C)}+2 \sum_{m=0}^{\delta}(-1)^{m} \hat{n}_{d}^{+}(m), \\
\chi_{r}^{d}=\sum_{\substack{C \in \mathcal{T a n}^{d}\left(J^{t}, \underline{x}^{t}\right) \\
T_{x_{1}^{t}} \mathbb{R} C=T_{1}}}(-1)^{m(C)}+2 \sum_{m=0}^{\delta}(-1)^{m} \hat{n}_{d}^{-}(m),
\end{gathered}
$$

where $\chi_{r+2}^{d}, \chi_{r}^{d}$ are invariants and $\hat{n}_{d}^{+}(m)$ (resp. $\left.\hat{n}_{d}^{-}(m)\right)$ is the number of real rational $J^{t}$-holomorphic curves of mass $m$ which pass through $\underline{x}^{t}$, realize the homology class $d$ and have a non isolated (resp. isolated) real double point at $x_{1}^{t}$. From the second part of Proposition 4.1, the term $\sum_{m=0}^{\delta}(-1)^{m} \hat{n}_{d}^{+}(m)$ decreases of $(-1)^{m\left(C^{t_{0}}\right)}$ as $t$ crosses the value $t_{0}$, while the term $\sum_{m=0}^{\delta}(-1)^{m} \hat{n}_{d}^{-}(m)$ increases of $(-1)^{m\left(C^{t_{0}}\right)+1}$. Since $\chi_{r+2}^{d}, \chi_{r}^{d}$ are constant, we deduce that

$$
\sum_{\substack{C \in \mathcal{T a n}^{d}\left(J^{t}, x^{t}\right) \\ T_{x_{1}^{t}} \mathbb{R} C=T_{1}}}(-1)^{m(C)}
$$

increases of $2(-1)^{m\left(C^{t_{0}}\right)}$ as $t$ crosses the value $t_{0}$, hence the result.

4.3. Proof of Theorem 0.1. - Choose a path $\gamma: t \in[0,1] \mapsto\left(J^{t}, \underline{x}^{t}\right) \in$ $\mathbb{R} \mathcal{J}_{\omega} \times \mathbb{R}_{\tau} X^{\nu}$ given by $\S 4$.1. The integer $\Gamma_{r}^{d}\left(J^{t}, \underline{x}^{t}\right)$ is then well defined for every $t \in[0,1]$ but a finite number of parameters $0<t_{0}<\cdots<t_{k}<1$ corresponding to accidents listed in $\S 4.1$. It is obviously constant between these parameters $t_{j}$, $j \in\{0, \ldots, k\}$, and we have to prove that is also does not change while crossing these parameters.

This is easy to check in cases 1$), 2), 3), \alpha), a), b), e$ ) listed in $§ 4.1$.

In cases 4$), d$ ), it follows from Proposition 4.2 .

The cases 5), $\gamma$ ),$c$ ) correspond to critical points which can be treated as in Proposition 2.16 of [11].

The case 6) follows from Proposition 2.7, the case 7) from Theorem 3.12 and cases 8), $\beta$ ) from Theorems 3.2 and 3.6.

Note that in this last case, the loss of two real cuspidal curves described by Theorem 3.2 is compensated by the decrease of the multiplicity of the corresponding reducible curve.

Cases $f$ ) and $\epsilon$ ) can be treated as in Proposition 2.14 of [11].

BULletin DE LA SOCiÉtÉ MATHÉmATiQUe DE FRANCE 
It only remains to prove the invariance in cases $\delta), g$ ). While crossing such a value $t_{k}$, the second term in the definition of $\Gamma_{r}^{d}\left(J^{t}, \underline{x}^{t}\right)$ remains clearly unchanged. Note that the number of real rational $J^{t}$-holomorphic curves which pass through $\left.\underline{x}^{t}, t \in\right] t_{k}-\epsilon, t_{k}+\epsilon\left[\backslash\left\{t_{k}\right\}\right.$, have $T_{i}$ as a tangent line at $x_{i}^{t}$ and degenerate onto a reducible curve $C^{t_{k}}$ having a double point at $x_{i}^{t_{k}}$ is at most 1 . Indeed, two such curves would intersect with multiplicity 2 at $x_{i}^{t_{k}}, 1$ at the other points of the configuration $\underline{x}^{t}$ and 2 near every double point of $C^{t_{k}}$ but $x_{i}^{t_{k}}$. This provides a total intersection index at least equal to

$$
d^{2}-c_{1}(X) d+2+c_{1}(X) d-3+2=d^{2}+1
$$

which is impossible. Since the mass of such a real rational curve which degenerate onto such a reducible curve $C^{t_{k}}$ is the one of $C^{t_{k}}$ and the parity of the number of real rational $J^{t}$-holomorphic curves which pass through $\underline{x}^{t}$, $t \in] t_{k}-\epsilon, t_{k}+\epsilon\left[\backslash\left\{t_{k}\right\}\right.$ and have $T_{i}$ as a tangent line at $x_{i}^{t}$ is independant of $t$, the result follows.

\section{BIBLIOGRAPHY}

[1] Barraud (J.-F.) - Courbes pseudo-holomorphes équisingulières en dimension 4, Bull. Soc. Math. France, t. 128 (2000), pp. 179-206.

[2] Hofer (H.), Lizan (V.) \& SikoraV (J.-C.) - On genericity for holomorphic curves in 4-dimensional almost-complex manifolds, J. Geom. Anal., t. 7 (1997), pp. 149-159.

[3] Ivashkovich (S.) \& Shevchishin (V.) - Structure of the moduli space in a neighborhood of a cusp-curve and meromorphic hulls, Invent. Math., t. 136 (1999), pp. 571-602.

[4] Kontsevich (M.) \& Manin (Y.) - Gromov-Witten classes, quantum cohomology, and enumerative geometry, pp. 607-653 in Mirror symmetry, II, in AMS/IP Stud. Adv. Math., vol. 1, Amer. Math. Society, Providence, RI, 1997.

[5] McDuff (D.) \& Salamon (D.) - J-holomorphic curves and symplectic topology, American Math. Soc. Colloquium Publications, vol. 52, Amer. Math. Society, Providence, RI, 2004.

[6] Shevchishin (V. V.) - Pseudoholomorphic curves and the symplectic isotopy problem, Preprint math.SG/0010262 (2000), Habilitation Thesis at Ruhr-University, Bochum, Germany.

[7] Siebert (B.) \& Tian (G.) - On the holomorphicity of genus 2 Lefschetz fibrations, Ann. of Math. (2), t. 161 (2005), pp. 959-1020.

[8] Sikorav (J.-C.) - Singularities of J-holomorphic curves, Math. Z., t. 226 (1997), pp. 359-373.

[9] WelschingeR (J.-Y.) - Towards relative invariants of real symplectic four-manifolds, Geom. Funct. Anal., To appear, see math.SG/0502358.

TOME $134-2006-\mathrm{N}^{\mathrm{O}} 2$ 
[10] _ Enumerative invariants of strongly semipositive real symplectic manifolds, Preprint math.AG/0509121 (2005).

[11] Invariants of real symplectic 4-manifolds and lower bounds in real enumerative geometry, Invent. Math., t. 162 (2005), pp. 195-234.

[12] Spinor states of real rational curves in real algebraic convex 3-manifolds and enumerative invariants, Duke Math. J., t. 127 (2005), pp. 89-121.

[13] Invariant count of holomorphic disks in the cotangent bundles of the two-sphere and real projective plane, Research announcement (2006). 